\title{
Lin's method for heteroclinic chains involving periodic orbits
}

\author{
Jürgen Knobloch ${ }^{1}$ and Thorsten Rieß ${ }^{2}$ \\ ${ }^{1}$ Faculty of Mathematics and Natural Sciences, Technische Universität Ilmenau, \\ PF 100565, 98684 Ilmenau, Germany \\ ${ }^{2}$ Center for Applied Mathematics, 657 Frank H.T. Rhodes Hall, Cornell University, \\ Ithaca, NY 14853, U.S.A. \\ E-mail: juergen.knobloch@tu-ilmenau.de, triess@cam.cornell.edu
}

\begin{abstract}
We present an extension of the theory known as Lin's method to heteroclinic chains that connect hyperbolic equilibria and hyperbolic periodic orbits. Based on the construction of a so-called Lin orbit, that is, a sequence of continuous partial orbits that only have jumps in a certain prescribed linear subspace, estimates for these jumps are derived. We use the jump estimates to discuss bifurcation equations for homoclinic orbits near heteroclinic cycles between an equilibrium and a periodic orbit (EtoP cycles).
\end{abstract}

AMS classification scheme numbers: 37C29, 37G25, 34C23, 34C60

\section{Introduction}

Connecting cycles involving hyperbolic equilibria and hyperbolic periodic orbits play an important role in many applications, cf. [12] and references therein. The bifurcation analysis in the vicinity of such a connecting cycle is crucial for the understanding of the system's behavior. In this respect, both the theoretical bifurcation analysis and numerical implementations are of high interest in current research.

Lin's method has proved to be an appropriate tool for discovering recurrent dynamics near a given cycle. The method dates from [14, where heteroclinic chains consisting of hyperbolic fixed points, all having the same index (dimension of the unstable manifold), and heteroclinic orbits connecting them are considered. The basic idea of Lin's method is to construct discontinuous orbits with well defined discontinuities (jumps), so-called Lin orbits, near the original cycle. By 'making these jumps zero' one finally finds real orbits staying for all time close to the cycle under consideration. In 1993 Sandstede [19] gained jump estimates which allow an effective discussion of the bifurcation equations. For a survey of the many applications and several extensions of the method we refer to [15]. 
In this paper we present an extension of the theory of Lin's method to arbitrary heteroclinic chains connecting hyperbolic equilibria and hyperbolic periodic orbits. Related problems have been studied in [5, 16, 17] and [18]; for the numerical implementation of these ideas we refer to [12].

In the presence of periodic orbits the construction of Lin orbits is much more involved, because the dynamics near the periodic orbit has to be incorporated. The handling of the flow near the periodic is the main difference between the approaches in [16, 17] and [18]. Based on the ideas in [18], we construct certain partial (discontinuous) orbits running between Poincaré sections of two consecutive periodic orbits of the given chain. Then the dynamics near each periodic orbit is described by means of the corresponding Poincaré map. Finally, the different orbits are coupled in the Poincaré section in each case. This approach allows to apply immediately results from Lin's method for discrete systems [10].

We consider a family of ODE

$$
\dot{x}=f(x, \lambda), f \in C^{k}\left(\mathbb{R}^{n} \times \mathbb{R}^{m}, \mathbb{R}^{n}\right), k \geq 3 .
$$

For a particular parameter value, say $\lambda=0$, we assume that the system has a heteroclinic chain consisting of hyperbolic periodic orbits $\gamma_{i}$ and heteroclinic orbits $q_{i}$ connecting $\gamma_{i}$ and $\gamma_{i+1}$. Here we explicitely admit that the minimal period of either of these periodic orbits may be zero, meaning that either of these orbits may be an equilibrium. We want to note that, for instance, a heteroclinic cycle between an equilibrium and a periodic orbit can be considered as such a heteroclinic chain. In this case the chain consists of copies of the cycle under consideration which are stringed together.

We refer to a segment $\gamma_{i} \cup q_{i} \cup \gamma_{i+1}$ of the given chain as a short heteroclinic chain segment. Near $q_{i}$ we construct a discontinuous orbit $X_{i}$ satisfying certain boundary conditions $\left(B_{i}^{-}\right)$near $\gamma_{i}$ and $\left(B_{i+1}^{+}\right)$near $\gamma_{i+1}$. There the discontinuity is a well defined jump $\Xi_{i}$ near $q_{i}(0)$. Those orbits we call short Lin orbit segments.

It can be shown that arbitrarily many consecutive short Lin orbit segments can be linked together to a Lin orbit close to the original chain, see [18] for chains related to heteroclinic cycles connecting one equilibrium and one periodic orbit. In the present paper we confine ourselves to linking two consecutive short Lin orbit segments $X_{l}$ and $X_{r}$ related to $\gamma_{l} \cup q_{l} \cup \gamma$ and $\gamma \cup q_{r} \cup \gamma_{r}$ to a long Lin orbit segment with boundary conditions $\left(B_{l}^{-}\right)$and $\left(B_{r}^{+}\right)$. Apart from the fact that this procedure reveals the basic idea for linking arbitrarily many consecutive short Lin orbit segments, it is eligible for consideration in its own right. So it suffices to consider long Lin orbit segments for the detection of 1-homoclinic orbits near a heteroclinic cycle connecting two periodic orbits. Here, 1-homoclinic orbits are characterized by only one large excursion before returning to their starting point.

If $\gamma$ is an equilibrium, the existence proof of long Lin orbit segments runs to large extent parallel to 'classical constructions' of Lin's method, see [18]. For that reason we consider only the case that $\gamma$ is a periodic orbit with nonzero minimal period. Roughly speaking, the orbits $X_{l}$ and $X_{r}$ are linked via an orbit $x$ that defines the behavior of 
the newly generated orbit along $\gamma$. We construct $x=x(y)$ as a suspension of a certain orbit $y$ of an appropriate Poincaré map. In this process the boundary conditions $\left(B_{l}^{-}\right)$ and $\left(B_{r}^{+}\right)$remain untouched.

Finally we give estimates of the jumps $\Xi_{l}$ and $\Xi_{r}$, which allow us to discuss the bifurcation equations $\Xi_{l}=0$ and $\Xi_{r}=0$ for detecting actual orbit segments near the given long orbit segment $\gamma_{l} \cup q_{l} \cup \gamma \cup q_{r} \cup \gamma_{r}$.

We apply our results to study homoclinic orbits near a heteroclinic cycle connecting a hyperbolic equilibrium $E$ and a hyperbolic periodic orbit $P$ (with nonzero minimal period), an EtoP cycle for short. Here we only consider 1-homoclinic orbits to the equilibrium. Those orbits may differ considerably in their length of stay near $P$. This length correlates to the number $\nu$ of rotations the homoclinic orbit performs along $P$ or, in the above notation, it correlates to the length $\nu$ of the orbit $y$.

Indeed, in numerical computations it has been observed that the homoclinic orbits for different $\nu$ all lie on the same continuation curve. Moreover, this continuation curve shows a certain snaking behavior and accumulates on a curve segment related to the existence of the primary EtoP cycle, cf. figure 5 panel (a). The addressed snaking behavior of a system with reinjection was revealed numerically in [11] and [12].

In particular, we explain two local phenomena appearing in this global snaking scenario. First we consider a codimension-one EtoP cycle. Apart from $E$ and $P$ this cycle consists of a robust heteroclinic orbit $q_{l}$ connecting $E$ to $P$, and a codimensionone heteroclinic orbit $q_{r}$ connecting $P$ to $E$. Further, the dimensions of the unstable manifold of $P$ and the stable manifold of $E$ add up to the space dimension.

Let $\lambda$ be the one-dimensional parameter unfolding $q_{r}$ and, hence, unfolding the entire cycle. In that unfolding we describe the accumulation of homoclinic orbits at the primary heteroclinic cycle. More precisely, we prove that there is a sequence $\left(\lambda_{\nu}\right)_{\nu \in \mathbb{N}}$ tending to zero such that for each $\lambda_{\nu}$ there is a homoclinic orbit to the equilibrium, while for $\lambda=0$ the heteroclinic cycle exists. Moreover, with increasing $\nu$ the corresponding homoclinic orbits stay longer near the periodic orbit, performing an increasing number of rotations along the periodic orbit.

In a second scenario we assume that $W^{u}(E)$ and $W^{s}(P)$ do no longer intersect transversally but have a quadratic tangency - still we assume that $q_{r}$ is of codimension one as described above. Then the entire EtoP cycle is of codimension two. Let the parameter $\lambda_{l / r}$ unfold the orbits $q_{l / r}$, and assume that the EtoP cycle exists for $\lambda=\left(\lambda_{l}, \lambda_{r}\right)=0$. Then, in the neighborhood of $\lambda=0$, we find a sequence of curves $\kappa_{\nu}$ in the $\lambda$-plane for which a homoclinic orbit to the equilibrium exists. As above with increasing $\nu$ the corresponding homoclinic orbits stay longer and longer near the periodic orbit, performing an increasing number of rotations along the periodic orbit. For each $\nu$ the curve $\kappa_{\nu}$ has a turning point $\lambda_{\nu}$ tending to zero as $\nu$ tends to infinity. This explains the curve progression of $h_{1}$ in a small neighborhood of $c_{1} \cap t_{0}$, cf. figure 5.

The paper is organized as follows. In section 2 we develop Lin's method for short heteroclinic chain segments. The main theorem in this respect is theorem 2.2, which states the existence of short Lin orbit segments. Corollary 2.8 extends theorem 2.2 to 
boundary conditions that enforce that the short Lin orbit segment 'starts' in the unstable manifold of $\gamma_{i}$. In lemma 2.10 we give an estimate of the jump function, which we extend in corollary 2.11 to the situation of corollary 2.8 . In section 3 we describe the coupling of two consecutive short Lin orbit segments $X_{l}$ and $X_{r}$ to a long Lin orbit segment. Their existence is stated in theorem 3.1 , and corollary 3.2 extends this assertion on $X_{l}$ and $X_{r}$ asymptotic to $\gamma_{l}$ and $\gamma_{r}$, respectively. The corresponding estimates of the jumps are given in lemma 3.11 and corollary 3.12. In section 4 we consider 1-homoclinic orbits near EtoP cycles. The corollaries 4.3 and 4.4 explain the accumulation of 1-homoclinic orbits, and corollary 4.7 describes the accumulation of vertices of continuation curves that are obtained by unfolding a ray (in parameter space) that is covered twice.

\section{Lin's method for short heteroclinic chains}

Consider the ODE (11). Throughout this section we assume that for $\lambda=0$ there is a short heteroclinic chain segment $\gamma^{-} \cup q \cup \gamma^{+}$with hyperbolic periodic orbits $\gamma^{-}$and $\gamma^{+}$. We explicitly admit that the minimal period $T^{-/+}$of either of them may be zero, meaning that $\gamma^{-}$and/or $\gamma^{+}$may be hyperbolic equilibria. Let $W_{\lambda}^{s / u}\left(\gamma^{ \pm}\right)$denote the stable/unstable manifolds of $\gamma^{ \pm}$, and we use the short notation $W^{s / u}\left(\gamma^{ \pm}\right)$for the corresponding manifolds at $\lambda=0$. Further, $T_{q} W^{s / u}$ denotes the tangent space of the corresponding manifold at $q$.

We introduce subspaces $W^{+}, W^{-}$and $U$ as follows:

$$
\begin{aligned}
& \left(T_{q(0)} W^{u}\left(\gamma^{-}\right) \cap T_{q(0)} W^{s}\left(\gamma^{+}\right)\right) \oplus W^{-}=T_{q(0)} W^{u}\left(\gamma^{-}\right), \\
& \left(T_{q(0)} W^{u}\left(\gamma^{-}\right) \cap T_{q(0)} W^{s}\left(\gamma^{+}\right)\right) \oplus W^{+}=T_{q(0)} W^{s}\left(\gamma^{+}\right) \text {and } \\
& \operatorname{span}\{f(q(0), 0)\} \oplus U=T_{q(0)} W^{u}\left(\gamma^{-}\right) \cap T_{q(0)} W^{s}\left(\gamma^{+}\right) .
\end{aligned}
$$

In other words, the linear spaces $W^{-}$and $W^{+}$are contained in the tangent spaces of the unstable and stable manifolds of $\gamma^{-}$and $\gamma^{+}$at $q(0)$, but do not contain their common directions, which are collected in $\operatorname{span}\{f(q(0), 0)\} \oplus U$.

Using a scalar product $\langle\cdot, \cdot\rangle$ in $\mathbb{R}^{n}$ we define

$$
Z:=\left(W^{+} \oplus W^{-} \oplus U \oplus \operatorname{span}\{f(q(0), 0)\}\right)^{\perp}
$$

and

$$
Y=W^{+} \oplus W^{-} \oplus U \oplus Z,
$$

and we denote the projection onto $U$ in accordance with the direct sum decomposition (3) by $P^{U}$. Note that either of the involved spaces $W^{ \pm}, U$ and $Z$ may be trivial. Finally, we define a cross-section $\Sigma$ of $q$ as

$$
\Sigma:=q(0)+Y \text {. }
$$

Our goal is to construct 'discontinuous orbits' near $q$ that satisfy certain boundary conditions $\left(B^{-}\right)$and $\left(B^{+}\right)$near $\gamma^{-}$and $\gamma^{+}$. Actually those orbits consist of two orbit segments where the end point of the first and the starting point of the second one are in $\Sigma$ and their difference is in $Z$, which is reflected in the boundary condition $(J)$. 
In a first step we prove the existence of orbit segments that lie in the unstable and stable manifolds of $\gamma^{-}$and $\gamma^{+}$, respectively, and that satisfy certain jump conditions in $\Sigma$.

Theorem 2.1. There is a constant $c>0$ such that for $|\lambda|<c$ and $u \in U,|u|<c$, there is a unique pair of solutions $\left(q^{-}(u, \lambda), q^{+}(u, \lambda)\right)$ of (11) that satisfy

(i) $q^{+}(u, \lambda)(0) \in W_{\lambda}^{s}\left(\gamma^{+}\right), q^{-}(u, \lambda)(0) \in W_{\lambda}^{u}\left(\gamma^{-}\right)$,

(ii) $q^{+}(u, \lambda)(0), q^{-}(u, \lambda)(0) \in \Sigma$,

(iii) $P^{U}\left(q^{+}(u, \lambda)(0)-q(0)\right)=P^{U}\left(q^{-}(u, \lambda)(0)-q(0)\right)=u$ and

(iv) $q^{+}(u, \lambda)(0)-q^{-}(u, \lambda)(0) \in Z$.

Figures 1 and 2 give a graphical interpretation of theorem 2.1, the proof is given in section 2.1.

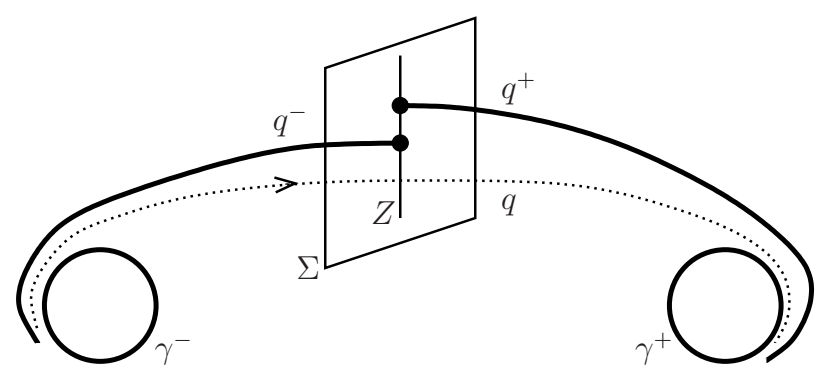

Figure 1. Sketch of the situation described in theorem 2.1, showing the orbits $q^{-} \subset W_{\lambda}^{u}\left(\gamma^{-}\right)$and $q^{+} \subset W_{\lambda}^{s}\left(\gamma^{+}\right)$. Within the cross-section $\Sigma$, the two orbits have a jump in the direction $Z$. Note that $\gamma^{-}$and $\gamma^{+}$are depicted as periodic orbits, but either of them may be an equilibrium. The dotted connection $q$ is present for $\lambda=0$.

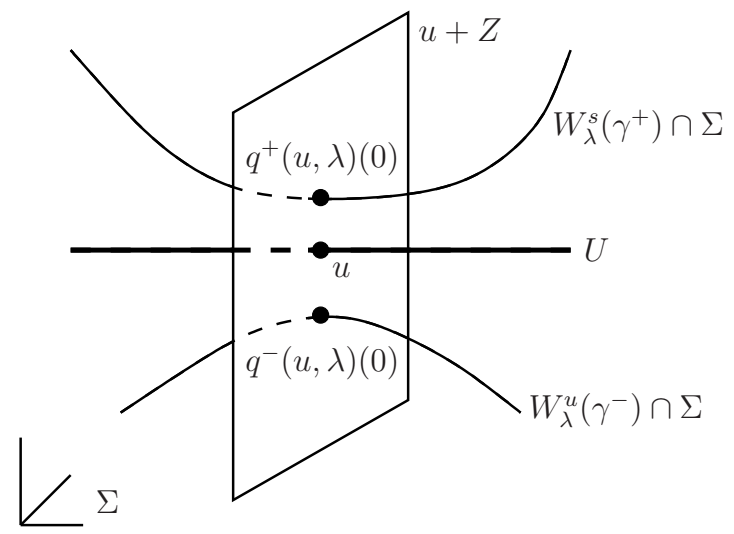

Figure 2. The situation inside $\Sigma$, depicted are the traces of $q^{-/+}(u, \lambda), W_{\lambda}^{u}\left(\gamma^{-}\right)$, $W_{\lambda}^{s}\left(\gamma^{+}\right)$and the direction $U$ consisting of the common tangent directions (restricted to $\Sigma$ ). The depicted situation corresponds to a 'quadratic tangency' of $W^{u}\left(\gamma^{-}\right)$and $W^{s}\left(\gamma^{+}\right)$. 
In the next step, we perturb the solutions $q^{ \pm}$given by theorem 2.1 to construct solutions that stay near the connecting orbit $q$ and satisfy projection boundary conditions near $\gamma^{-}$and $\gamma^{+}$. Moreover, these solutions are also allowed to have a jump in the direction $Z$ (within $\Sigma$ ).

To formulate the boundary conditions we define projections $P^{ \pm}(u, \lambda)(t)$ by

$$
\begin{aligned}
& \operatorname{im} P^{+}(u, \lambda)(0)=T_{q^{+}(u, \lambda)(0)} W_{\lambda}^{s}\left(\gamma^{+}\right), \\
& \operatorname{ker} P^{+}(u, \lambda)(0)=W^{-} \oplus Z \text { and } \\
& P^{+}(u, \lambda)(t) \quad:=\Phi^{+}(t, 0)\left(P^{+}(u, \lambda)(0)\right) \Phi^{+}(0, t), t \in \mathbb{R}^{+},
\end{aligned}
$$

and analogously

$$
\begin{aligned}
& \operatorname{im} P^{-}(u, \lambda)(0)=T_{q^{-}(u, \lambda)(0)} W_{\lambda}^{u}\left(\gamma^{-}\right) \\
& \operatorname{ker} P^{-}(u, \lambda)(0)=W^{+} \oplus Z \text { and } \\
& P^{-}(u, \lambda)(t) \quad:=\Phi^{-}(t, 0)\left(P^{-}(u, \lambda)(0)\right) \Phi^{-}(0, t), t \in \mathbb{R}^{-} .
\end{aligned}
$$

Here $\Phi^{ \pm}(\cdot, \cdot)=\Phi^{ \pm}(u, \lambda)(\cdot, \cdot)$ denotes the transition matrix of the variational equation along $q^{ \pm}(u, \lambda)(\cdot)$.

Throughout we denote by $|\cdot|$ the absolute value of a number or the the Euclidian norm of an $n$-tuple. For elements $a=\left(a^{-}, a^{+}\right) \in \mathbb{R}^{n} \times \mathbb{R}^{n}$ we define $\|a\|:=$ $\max \left\{\left|a^{-}\right|,\left|a^{+}\right|\right\}$.

Theorem 2.2. Fix $\omega^{-}, \omega^{+}>0$. There is a constant $c>0$ such that for $|\lambda|<c, u \in U$, $|u|<c$, and $a=\left(a^{-}, a^{+}\right) \in \mathbb{R}^{n} \times \mathbb{R}^{n},\|a\|<c$, there is a unique pair of solutions $\left(x^{-}, x^{+}\right)$of (11) that satisfies

$(J) x^{-/+}(a, u, \lambda)(0) \in \Sigma, \quad x^{-}(a, u, \lambda)(0)-x^{+}(a, u, \lambda)(0) \in Z$,

$\left(B^{-}\right)\left(i d-P^{-}(u, \lambda)\left(-\omega^{-}\right)\right)\left(x^{-}(a, u, \lambda)\left(-\omega^{-}\right)-q^{-}(u, \lambda)\left(-\omega^{-}\right)-a^{-}\right)=0$,

$\left(B^{+}\right)\left(i d-P^{+}(u, \lambda)\left(\omega^{+}\right)\right)\left(x^{+}(a, u, \lambda)\left(\omega^{+}\right)-q^{+}(u, \lambda)\left(\omega^{+}\right)-a^{+}\right)=0$.

Moreover, $\left(x^{-}, x^{+}\right)=\left(x^{-}, x^{+}\right)(a, u, \lambda)$ depends smoothly on $(a, u, \lambda)$.

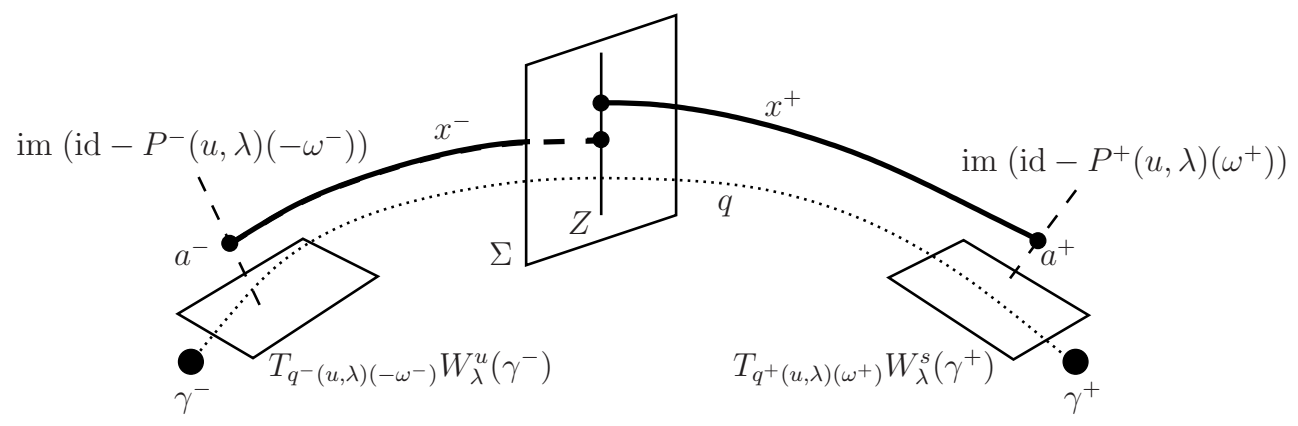

Figure 3. Sketch of a short Lin orbit segment $\left(x^{-}, x^{+}\right)$near a short heteroclinic chain segment $\gamma^{-} \cup q \cup \gamma^{+}$.

In other words, theorem 2.2 states the existence of short Lin orbits segments $X=\left(x^{-}, x^{+}\right)$with boundary conditions $\left(B^{-}\right)$and $\left(B^{+}\right)$for sufficiently small $(a, u, \lambda)$, for a sketch of this situation see figure 3. Note that $X$ also depends on $\omega^{-}, \omega^{+}>0$, which we suppress from our notation in this section. The proof is given in section 2.2 . 


\subsection{Orbits in the stable/unstable manifolds - the proof of theorem 2.1}

From a geometrical point of view, the statement of theorem 2.1 is rather clear. Hence we give a proof which exploits the geometry of the traces of the involved manifolds in $\Sigma$, see figure 2. By $B_{X}(x, r)$ we denote a closed ball in $X$ centered at $x$ with radius $r$. If the space $X$ is clear from the context we will also write $B(x, r)$ for short.

Proof of theorem 2.1. Using the direct sum decomposition (3) we find the following representations of the traces in $\Sigma$ of the stable/unstable manifolds of $\gamma^{-/+}$locally around $q(0)$ : For $\varepsilon$ sufficiently small and for $w^{s} \in W_{\lambda}^{s}\left(\gamma^{+}\right) \cap \Sigma \cap B(q(0), \varepsilon)$ there are smooth functions $\tilde{w}^{-}: W^{+} \times U \times \mathbb{R}^{m} \rightarrow W^{-}$with $\tilde{w}^{-}(0,0,0)=0, D_{1} \tilde{w}^{-}(0,0,0)=0$ and $z^{+}: W^{+} \times U \times \mathbb{R}^{m} \rightarrow Z$ such that

$$
w^{s}=q(0)+w^{+}+\tilde{w}^{-}\left(w^{+}, u^{+}, \lambda\right)+z^{+}\left(w^{+}, u^{+}, \lambda\right)+u^{+} .
$$

Similarly for $w^{u} \in W_{\lambda}^{u}\left(\gamma^{-}\right) \cap \Sigma \cap B(q(0), \varepsilon)$ there are smooth functions $\tilde{w}^{+}: W^{-} \times U \times$ $\mathbb{R}^{m} \rightarrow W^{+}$with $\tilde{w}^{+}(0,0,0)=0, D_{1} \tilde{w}^{+}(0,0,0)=0$ and $z^{-}: W^{-} \times U \times \mathbb{R}^{m} \rightarrow Z$ such that

$$
w^{u}=q(0)+\tilde{w}^{+}\left(w^{-}, u^{-}, \lambda\right)+w^{-}+z^{-}\left(w^{-}, u^{-}, \lambda\right)+u^{-} .
$$

The demand that $q^{+}(u, \lambda)(0)-q^{-}(u, \lambda)(0) \in Z$ results in $u^{-}=u^{+}=: u$ and

$$
\begin{aligned}
& w^{+}=\tilde{w}^{+}\left(w^{-}, u, \lambda\right), \\
& w^{-}=\tilde{w}^{-}\left(w^{+}, u, \lambda\right),
\end{aligned}
$$

which then can be solved for $\left(w^{+}, w^{-}\right)=\left(\hat{w}^{+}(u, \lambda), \hat{w}^{-}(u, \lambda)\right)$ around $(u, \lambda)=(0,0)$.

Now we get $q^{+}(u, \lambda)$ as the solution of the initial the value problem

$$
\begin{aligned}
& \dot{x} \quad=f(x, \lambda) \\
& x(0)=q(0)+\hat{w}^{+}(u, \lambda)+\tilde{w}^{-}\left(\hat{w}^{+}(u, \lambda), u, \lambda\right)+z^{+}\left(\hat{w}^{+}(u, \lambda), u, \lambda\right)+u,
\end{aligned}
$$

and $q^{+}(u, \lambda)$ is the solution of the initial the value problem

$$
\begin{aligned}
\dot{x} & =f(x, \lambda) \\
x(0) & =q(0)+\tilde{w}^{+}\left(\hat{w}^{-}(u, \lambda), u, \lambda\right)+\hat{w}^{-}(u, \lambda)+z^{-}\left(\hat{w}^{-}(u, \lambda), u, \lambda\right)+u .
\end{aligned}
$$

\subsection{Short Lin orbit segments - the proof of theorem 2.2}

In this section we give a detailed proof of theorem 2.2. This proof is based on the ideas of Lin's method, but used in a slightly different way. The main difference to the 'classical' proof of Lin's method is that we keep the boundary conditions near $\gamma^{-}$and $\gamma^{+}$as linear projection conditions, while finding solutions of the full nonlinear system that additionally satisfy certain jump conditions.

We start with a lemma that provides some properties of the projections $P^{ \pm}(u, \lambda)(\cdot)$, as introduced in (44) and (5), that are used in the proofs throughout this section. 
Lemma 2.3. There are projections $P_{s}^{+}(u, \lambda)(\cdot)$ and $P_{c}^{+}(u, \lambda)(\cdot)$ such that

$$
P^{+}(u, \lambda)(t)=P_{s}^{+}(u, \lambda)(t)+P_{c}^{+}(u, \lambda)(t) \text { for } t \in \mathbb{R}^{+} .
$$

The projections $P_{s / c}^{+}(u, \lambda)(\cdot)$ satisfy the following:

(i) $\Phi(t, \tau) P_{s / c}^{+}(\tau)=P_{s / c}^{+}(t) \Phi(t, \tau) \quad \forall t, \tau \in \mathbb{R}^{+}$,

(ii) there are constants $K>0, \delta^{s}, \delta^{u}>\delta^{c} \geq 0$ such that

$$
\begin{array}{ll}
\left|\Phi(t, \tau) P_{s}^{+}(\tau)\right| \leq K e^{-\delta^{s}(t-\tau)} & , t \geq \tau, \\
\left|\Phi(t, \tau) P_{c}^{+}(\tau)\right| \leq K e^{\delta^{c}(t-\tau)} & , t \geq \tau, \\
\left|\Phi(t, \tau) P_{c}^{+}(\tau)\right| \leq K e^{-\delta^{c}(t-\tau)} & , \tau \geq t, \\
\left|\Phi(t, \tau)\left(i d-P^{+}(\tau)\right)\right| \leq K e^{-\delta^{u}(\tau-t)}, \tau \geq t .
\end{array}
$$

This lemma follows immediately from the fact that the variational equation along the solutions $q^{+}(u, \lambda)$ has an exponential trichotomy on $\mathbb{R}^{+}$[3, 9, 6]. Note that the exponents $\delta^{s / c / u}$ are determined by the eigenvalues/Floquet exponents of $\gamma^{+}$. Since $\gamma^{+}$ is a hyperbolic periodic orbit, we have $\delta^{c}=0$ [3]. We want to note explicitely that the images of $P_{s}^{+}(u, \lambda)(t)$ are well-determined - these are the tangent spaces of the strong stable fiber of $\gamma^{+}$at $q^{+}(u, \lambda)(t)$. Also note that if $\gamma^{+}$is a hyperbolic equilibrium, the variational equation along $q^{+}(u, \lambda)$ has in fact an exponential dichotomy [7], i.e. $P_{c}^{+}(u, \lambda)=0$, and $\operatorname{im} P_{s}^{+}(u, \lambda)(t)=T_{q^{+}(u, \lambda)(t)} W_{\lambda}^{s}\left(\gamma^{+}\right)$.

For the projection $P^{-}(u, \lambda)$ an analogous lemma holds, exploiting that the variational equation along the solutions $q^{-}(u, \lambda)$ has an exponential trichotomy on $\mathbb{R}^{-}$:

$$
P^{-}(u, \lambda)(t)=P_{u}^{-}(u, \lambda)(t)+P_{c}^{-}(u, \lambda)(t) \text { for } t \in \mathbb{R}^{-},
$$

where $P_{u}^{-}(u, \lambda)(t)$ projects on the tangent space of the strong unstable fiber at $q^{-}(u, \lambda)(t)$.

To prove theorem 2.2 we consider small perturbations of the solutions $q^{-/+}(u, \lambda)$ :

$$
\begin{array}{ll}
x^{-}(t):=q^{-}(u, \lambda)(t)+v^{-}(t), & t \in \mathbb{R}^{-}, \\
x^{+}(t):=q^{+}(u, \lambda)(t)+v^{+}(t), & t \in \mathbb{R}^{+} .
\end{array}
$$

The perturbation terms $v^{-}$and $v^{+}$are solutions of

$$
\begin{aligned}
& \dot{v}^{-}=D_{1} f\left(q^{-}(u, \lambda)(t), \lambda\right) v^{-}+h^{-}\left(t, v^{-}, u, \lambda\right), \\
& \dot{v}^{+}=D_{1} f\left(q^{+}(u, \lambda)(t), \lambda\right) v^{+}+h^{+}\left(t, v^{+}, u, \lambda\right),
\end{aligned}
$$

where $h^{ \pm}(t, v, u, \lambda):=f\left(q^{ \pm}(u, \lambda)(t)+v, \lambda\right)-f\left(q^{ \pm}(u, \lambda)(t), \lambda\right)-D_{1} f\left(q^{ \pm}(u, \lambda)(t), \lambda\right)$. The boundary conditions $(J)$ and $\left(B^{ \pm}\right)$for $x^{ \pm}$yield boundary conditions for $v^{ \pm}$:

$\left(J_{v}\right) v^{ \pm}(0) \in W^{-} \oplus W^{+} \oplus Z, \quad v^{-}(0)-v^{+}(0) \in Z$,

$\left(B_{v}^{-}\right)\left(\mathrm{id}-P^{-}(u, \lambda)\left(-\omega^{-}\right)\right) v^{-}\left(-\omega^{-}\right)=\left(\mathrm{id}-P^{-}(u, \lambda)\left(-\omega^{-}\right)\right) a^{-}$,

$\left(B_{v}^{+}\right)\left(\mathrm{id}-P^{+}(u, \lambda)\left(\omega^{+}\right)\right) v^{+}\left(\omega^{+}\right)=\left(\mathrm{id}-P^{+}(u, \lambda)\left(\omega^{+}\right)\right) a^{+}$.

In a first approximation of (17), we replace the functions $h^{-}$and $h^{+}$by functions $g^{-}$ and $g^{+}$only depending on $t$ :

$$
\begin{aligned}
& \dot{v}^{-}=D_{1} f\left(q^{-}(u, \lambda)(t), \lambda\right) v^{-}+g^{-}(t), \\
& \dot{v}^{+}=D_{1} f\left(q^{+}(u, \lambda)(t), \lambda\right) v^{+}+g^{+}(t) .
\end{aligned}
$$


For given $\omega^{-}$and $\omega^{+}$we write $\omega:=\left(\omega^{-}, \omega^{+}\right)$, and we introduce the space $V_{\boldsymbol{\omega}}$ of pairs of continuous functions as

$$
V_{\boldsymbol{\omega}}:=\left\{\left(v^{-}, v^{+}\right): v^{-} \in C\left(\left[-\omega^{-}, 0\right], \mathbb{R}^{n}\right) \text { and } v^{+} \in C\left(\left[0, \omega^{+}\right], \mathbb{R}^{n}\right)\right\} .
$$

We equip $V_{\boldsymbol{\omega}}$ with the norm $\left\|\left(v^{-}, v^{+}\right)\right\|:=\max \left\{\left\|v^{-}\right\|,\left\|v^{+}\right\|\right\}$, where $\left\|v^{ \pm}\right\|$denotes the supremum norm.

We actually perform the proof of theorem 2.2 in two steps. First we consider the 'linearized' equation (8) (linearized in the sense that $g^{ \pm}$does not depend on $v^{ \pm}$) and show that there are unique solutions $\hat{v}^{ \pm}$satisfying boundary conditions $\left(J_{v}\right),\left(B_{v}^{-}\right)$and $\left(B_{v}^{+}\right)$; see Lemma 2.4 below. Of course $\hat{v}^{ \pm}$depend (among others) on $g^{ \pm}$. In the next step we replace the function $g^{ \pm}$in these dependencies by $h^{ \pm}$. This gives a fixed point equation (see (18) below) that is equivalent to (7) with boundary conditions $\left(J_{v}\right),\left(B_{v}^{-}\right)$ and $\left(B_{v}^{+}\right)$.

Lemma 2.4. Let $u$ and $\lambda$ be in accordance with theorem 2.1, and let $\boldsymbol{\omega}$ be fixed. Then, for given $a=\left(a^{-}, a^{+}\right) \in \mathbb{R}^{n} \times \mathbb{R}^{n}$ and $g=\left(g^{-}, g^{+}\right) \in V_{\boldsymbol{\omega}}$ there is a unique pair of solutions $\hat{v}=\left(\hat{v}^{-}, \hat{v}^{+}\right) \in V_{\boldsymbol{\omega}}, \hat{v}=\hat{v}(g, a, u, \lambda)$, of (8) that satisfy boundary conditions $\left(J_{v}\right),\left(B_{v}^{-}\right)$and $\left(B_{v}^{+}\right)$.

For fixed $u$ and $\lambda$ the pair of solutions $\hat{v}$ depends linearly on $(g, a)$, and it depends smoothly on $(g, a, u, \lambda)$. Moreover, there are constants $\hat{C}_{a}, \hat{C}_{g}>0$ such that

$$
\|\hat{v}(g, a, u, \lambda)\| \leq \hat{C}_{a}\|a\|+\hat{C}_{g}\|g\| .
$$

The constant $\hat{C}_{a}$ is uniform in $\omega^{+}$and $\omega^{-}$, while $\hat{C}_{g}$ is uniform in $\omega^{ \pm}$only if $\gamma^{ \pm}$is an equilibrium.

Proof. For a shorter notation in this proof we omit the dependencies of $\Phi$ and $P^{ \pm}$on $u$ and $\lambda$. The variation of constants formula gives

$$
\begin{aligned}
& v^{-}(t)=\Phi^{-}(t, 0) v^{-}(0)+\int_{0}^{t} \Phi^{-}(t, \tau) g^{-}(\tau) d \tau, \\
& v^{+}(t)=\Phi^{+}(t, 0) v^{+}(0)+\int_{0}^{t} \Phi^{+}(t, \tau) g^{+}(\tau) d \tau
\end{aligned}
$$

as solutions of (8), which can be transformed to

$$
\begin{aligned}
\left(\mathrm{id}-P^{-}(0)\right) v^{-}(0)= & \Phi^{-}\left(0,-\omega^{-}\right)\left(\mathrm{id}-P^{-}\left(-\omega^{-}\right)\right) v^{-}\left(-\omega^{-}\right) \\
& +\int_{-\omega^{-}}^{0} \Phi^{-}(0, \tau)\left(\mathrm{id}-P^{-}(\tau)\right) g^{-}(\tau) d \tau \\
\left(\mathrm{id}-P^{+}(0)\right) v^{+}(0)=\Phi^{+}\left(0, \omega^{+}\right)\left(\mathrm{id}-P^{+}\left(\omega^{+}\right)\right) v^{+}\left(\omega^{+}\right) & -\int_{0}^{\omega^{+}} \Phi^{+}(0, \tau)\left(\mathrm{id}-P^{+}(\tau)\right) g^{+}(\tau) d \tau .
\end{aligned}
$$


Thus

$$
\begin{aligned}
\left(\mathrm{id}-P^{-}(0)\right) v^{-}(0)= & \Phi^{-}\left(0,-\omega^{-}\right)\left(\mathrm{id}-P^{-}\left(-\omega^{-}\right)\right) a^{-} \\
& +\int_{-\omega^{-}}^{0} \Phi^{-}(0, \tau)\left(\mathrm{id}-P^{-}(\tau)\right) g^{-}(\tau) d \tau, \\
\left(\mathrm{id}-P^{+}(0)\right) v^{+}(0)= & \Phi^{+}\left(0, \omega^{+}\right)\left(\mathrm{id}-P^{+}\left(\omega^{+}\right)\right) a^{+} \\
& -\int_{0}^{\omega^{+}} \Phi^{+}(0, \tau)\left(\mathrm{id}-P^{+}(\tau)\right) g^{+}(\tau) d \tau .
\end{aligned}
$$

We decompose $v^{-}(0), v^{+}(0)$ in accordance with the boundary condition $\left(J_{\nu}\right)$

$$
\begin{aligned}
& v^{-}(0)=w^{-}+w^{+}+z^{-}, \\
& v^{+}(0)=w^{-}+w^{+}+z^{+},
\end{aligned}
$$

where $w^{-} \in W^{-}, w^{+} \in W^{+}$and $z^{ \pm} \in Z$.

With that the left-hand side of (11) can be considered as a linear mapping

$$
\begin{aligned}
L: W^{+} \times W^{-} \times Z \times Z & \rightarrow\left(W^{+} \oplus Z\right) \times\left(W^{-} \oplus Z\right) \\
\left(w^{+}, w^{-}, z^{+}, z^{-}\right) & \mapsto \quad\left(\begin{array}{c}
\left(w^{+}+z^{-}\right) \\
\left(w^{-}+z^{+}\right)
\end{array}\right),
\end{aligned}
$$

which is invertible. Hence we can solve (11) for $v^{+}(0), v^{-}(0)$ linearly depending on $(g, a)$. Incorporating the dependence on $(u, \lambda)$ finally gives a solution $\hat{v}=\hat{v}(g, a, u, \lambda)$ of (8) satisfying the boundary conditions $\left(J_{\nu}\right),\left(B_{\nu}^{-}\right)$and $\left(B_{\nu}^{+}\right)$. Note that $\hat{v}$ depends linearly on $(g, a)$, and smoothly on $(g, a, u, \lambda)$.

To prove estimate (9), we decompose $\hat{v}^{+}$by means of the projection $P^{+}$:

$$
\hat{v}^{+}(t)=\left(\mathrm{id}-P^{+}(t)\right) \hat{v}^{+}(t)+P^{+}(t) \hat{v}^{+}(t) .
$$

Thus we have

$$
\left|\hat{v}^{+}(t)\right| \leq\left|\left(\mathrm{id}-P^{+}(t)\right) \hat{v}^{+}(t)\right|+\left|P^{+}(t) \hat{v}^{+}(t)\right| .
$$

We use the variation of constants formula and the estimates of lemma 2.3 to derive an estimate for the second term of (12):

$$
\begin{aligned}
\left|P^{+}(t) \hat{v}^{+}(t)\right| & =\left|P^{+}(t)\left(\Phi^{+}(t, 0) \hat{v}^{+}(0)+\int_{0}^{t} \Phi^{+}(t, \tau) g^{+}(\tau) d \tau\right)\right| \\
& \leq K\left(e^{-\delta^{s} t}+e^{\delta^{c} t}\right)\left|\hat{v}^{+}(0)\right|+M\left\|g^{+}\right\| \\
& \leq K\left(e^{-\delta^{s} t}+1\right)\left|\hat{v}^{+}(0)\right|+M\left\|g^{+}\right\| .
\end{aligned}
$$

The constants $\delta^{s}, \delta^{c}$ and $K$ are the corresponding constants of the exponential trichotomy $\left(\delta^{s}>\delta^{c}=0\right.$; see lemma 2.3 and the remarks above). Note that if $\gamma^{+}$ is not an equilibrium, the constant $M$ depends on $\omega^{+}$, in fact $M \rightarrow \infty$ as $\omega^{+} \rightarrow \infty$ in the same order as $\omega^{+}$.

We estimate $\left|\hat{v}^{+}(0)\right|$ by applying $L^{-1}$ to (11) and using lemma 2.3 once again:

$$
\left|\hat{v}^{+}(0)\right| \leq\left\|L^{-1}\right\| \tilde{K}\left(\left|a^{+}\right|+\left|a^{-}\right|\right)+\hat{M}\left\|\left(g^{+}, g^{-}\right)\right\| .
$$


Heteroclinic chains involving periodic orbits

Here, the constant $\hat{M}$ is uniform in $\omega^{+}$and $\omega^{-}$.

Thus we have

$$
\left.\left|P^{+}(t) v^{+}(t)\right| \leq C_{1, a}\|a\|+C_{1, g}\|g\|\right) .
$$

The constant $C_{1, a}$ is uniform in $\omega^{+}$and $\omega^{-}$, while $C_{1, g}(\boldsymbol{\omega})$ tends to infinity in the same order as $\|\boldsymbol{\omega}\|$.

For the first term of the right hand side of (12) we use

$$
\begin{aligned}
\left(\mathrm{id}-P^{+}(t)\right) \hat{v}^{+}(t)=\Phi^{+} & \left(t, \omega^{+}\right)\left(\mathrm{id}-P^{+}\left(\omega^{+}\right)\right) a^{+} \\
& \quad-\int_{t}^{\omega^{+}} \Phi^{+}(t, \tau)\left(\mathrm{id}-P^{+}(\tau)\right) g^{+}(\tau) d \tau
\end{aligned}
$$

and thus finally get

$$
\left|\left(\mathrm{id}-P^{+}(t)\right) \hat{v}^{+}(t)\right| \leq C_{2}\left(\left|a^{+}\right|+\|g\|\right) .
$$

Note that $C_{2}$ is uniform in $\omega^{+}$(and does not depend on $\omega^{-}$).

Summarizing, there are constants $\hat{C}_{a}^{+}$and $\hat{C}_{g}^{+}$such that

$$
\left\|\hat{v}^{+}(g, a, u, \lambda)\right\| \leq \hat{C}_{a}^{+}\|a\|+\hat{C}_{g}^{+}\|g\|,
$$

where $\hat{C}_{a}^{+}$is uniform in $\omega^{ \pm}$and $\hat{C}_{g}^{+}(\boldsymbol{\omega})$ tends to infinity in the same order as $\|\boldsymbol{\omega}\|$. Proceeding with $\hat{v}^{-}$in a similar way yields estimate (9).

Lemma 2.5. Let the assumption of lemma 2.4 hold. We define functions

$$
\begin{aligned}
& \hat{a}_{\perp}^{+}(g, a, u, \lambda):=P_{s}^{+}(u, \lambda)\left(\omega^{+}\right) \hat{v}^{+}(g, a, u, \lambda)\left(\omega^{+}\right), \\
& \hat{a}_{\perp}^{-}(g, a, u, \lambda):=P_{u}^{-}(u, \lambda)\left(-\omega^{-}\right) \hat{v}^{-}(g, a, u, \lambda)\left(-\omega^{-}\right) .
\end{aligned}
$$

There are constants $\delta^{s}, \delta^{u}>0$ and $\hat{C}>0$ such that

$$
\left|\hat{a}_{\perp}^{+}\right| \leq \hat{C}\left(e^{-\delta^{s} \omega^{+}}\|a\|+\|g\|\right), \quad\left|\hat{a}_{\perp}^{-}\right| \leq \hat{C}\left(e^{-\delta^{u} \omega^{-}}\|a\|+\|g\|\right) .
$$

For the derivatives of $\hat{a}_{\perp}^{+}$and $\hat{a}_{\perp}^{-}$the following estimates hold:

$$
\left\|D_{2} \hat{a}_{\perp}^{+}(g, a, u, \lambda)\right\| \leq \hat{C} e^{-\delta^{s} \omega^{+}}, \quad\left\|D_{2} \hat{a}_{\perp}^{-}(g, a, u, \lambda)\right\| \leq \hat{C} e^{-\delta^{u} \omega^{-}}
$$

and

$$
\left\|D_{1} \hat{a}_{\perp}^{+}(g, a, u, \lambda)\right\|,\left\|D_{1} \hat{a}_{\perp}^{-}(g, a, u, \lambda)\right\| \leq \hat{C} .
$$

Proof. For estimate (14) we use lemma 2.3 again:

$$
\begin{aligned}
\left|\hat{a}_{\perp}^{+}(g, a, u, \lambda)\right| & =\left|P_{s}^{+}\left(\omega^{+}\right)\left(\Phi^{+}\left(\omega^{+}, 0\right) \hat{v}^{+}(0)+\int_{0}^{\omega^{+}} \Phi^{+}\left(\omega^{+}, \tau\right) g^{+}(\tau) d \tau\right)\right| \\
& \leq K e^{-\delta^{s} \omega^{+}}\left|\hat{v}^{+}(0)\right|+\hat{M}\left\|g^{+}\right\| \\
& \leq C\left(e^{-\delta^{s} \omega^{+}}\|a\|+\|g\|\right) .
\end{aligned}
$$

For the derivative we note that the dependencies of $\hat{v}^{ \pm}$on $(g, a)$ are linear. Hence, there are linear operators $\tilde{L}^{ \pm}$depending on $u$ and $\lambda$ such that $\hat{v}^{ \pm}(g, a, u, \lambda)=$ $\tilde{L}^{ \pm}(u, \lambda)(g, a)=\tilde{L}^{ \pm}(u, \lambda)(g, 0)+\tilde{L}^{ \pm}(u, \lambda)(0, a)$. Due to their definition, $\hat{a}_{\perp}^{ \pm}$also depend linearly on $(g, a)$. Thus the estimates (15) and (16) follow. 
Now we look for solutions of (77) satisfying the boundary conditions $\left(J_{v}\right),\left(B_{v}^{-}\right)$and $\left(B_{v}^{+}\right)$. For that purpose, in $\hat{v}(g, a, u, \lambda)$ we formally replace the function $g$ by $h$. To perform this substitution we define the Nemytskii operator $H:=\left(H^{-}, H^{+}\right)$:

$$
\begin{aligned}
H: V_{\boldsymbol{\omega}} \times U \times \mathbb{R}^{m} & \rightarrow \quad V_{\boldsymbol{\omega}} \\
(v, u, \lambda) & \mapsto\left(H^{-}\left(v^{-}, u, \lambda\right), H^{+}\left(v^{+}, u, \lambda\right)\right),
\end{aligned}
$$

where

$$
H^{-}(v, u, \lambda)(\cdot):=h^{-}(\cdot, v(\cdot), u, \lambda), \quad H^{+}(v, u, \lambda)(\cdot):=h^{+}(\cdot, v(\cdot), u, \lambda) .
$$

In [19] it is verified that $H$ has the stated mapping properties and that $H$ is smooth.

Summarizing, we find that a function $v$ solves the boundary value problem (17), $\left.\left(J_{v}\right),\left(B_{v}^{-}\right),\left(B_{v}^{+}\right)\right)$if and only if it satisfies the following fixed point equation in $V_{\boldsymbol{\omega}}$ :

$$
v=\hat{v}(H(v, u, \lambda), a, u, \lambda)=: F(v, a, u, \lambda) .
$$

Note that

$$
F: V_{\boldsymbol{\omega}} \times\left(\mathbb{R}^{n} \times \mathbb{R}^{n}\right) \times U \times \mathbb{R}^{m} \rightarrow V_{\boldsymbol{\omega}} .
$$

Lemma 2.6. Fix some $\boldsymbol{\omega}$. There are functions $\bar{\varepsilon}, \tilde{c}, \bar{c}, \Omega: \mathbb{R} \rightarrow \mathbb{R}^{+}$such that for all $K>1$ the fixed point problem (18) has a unique solution $v=\left(v^{-}, v^{+}\right) \in V_{\boldsymbol{\omega}}$, $v=v(a, u, \lambda)$, in an $\bar{\varepsilon}(K)$-neighborhood of $0 \in V_{\boldsymbol{\omega}}$, provided that $|\lambda|,|u|<\bar{c}(K)$, and $\|a\|<\tilde{c}(K)$. The solution $v$ depends smoothly on $(a, u, \lambda)$.

Proof. We use the Banach fixed point theorem to prove existence and uniqueness of $v$. First we show that there is an $F$-invariant closed ball $B(0, \bar{\varepsilon}) \subset V_{\boldsymbol{\omega}}$ and then that $F$ is a contraction on $B(0, \bar{\varepsilon})$ with respect to $v$.

Let $\hat{C}_{a}, \hat{C}_{g}$ and $\hat{C}$ be the constants in accordance with lemma 2.4 and lemma 2.5, respectively. Define $C:=\max \left\{1, \hat{C}, \hat{C}_{a}, \hat{C}_{g}\right\}$. Then, according to (9),

$$
\|F(v, a, u, \lambda)\| \leq C(\|a\|+\|H(v, u, \lambda)\|) .
$$

We start with an estimate for $\|H(v, u, \lambda)\|$. From the definition of $h^{ \pm}$we see that $H(0,0,0)=0$ and thus we can use the mean value theorem to get the estimate

$$
\begin{array}{rl}
\|H(v, u, \lambda)\| \leq \int_{0}^{1} \| D_{1} & H(s(v, u, \lambda))\|d s\| v \| \\
& +\int_{0}^{1}\left\|D_{2} H(s(v, u, \lambda))\right\| d s|u| \\
& +\int_{0}^{1}\left\|D_{3} H(s(v, u, \lambda))\right\| d s|\lambda| .
\end{array}
$$

Applying the mean value theorem to $D_{1} H$ we find that there is an appropriate constant $D>0$ such that with

$$
\bar{\varepsilon}(K):=\frac{1}{K^{2} C^{2} D}
$$

the following holds: If $|\lambda|,\|v\|,|u|<\bar{\varepsilon}(K)$ then, since $D_{1} H(0,0,0)=0$,

$$
\left\|D_{1} H(v, u, \lambda)\right\| \leq \frac{1}{7 K^{2} C^{2}} .
$$


Heteroclinic chains involving periodic orbits

Further, there is a constant $E$ such that for all $v, u$ and $\lambda$ taken from some neighborhood of the origin

$$
\int_{0}^{1}\left\|D_{2} H(s(v, u, \lambda))\right\| d s<E, \quad \int_{0}^{1}\left\|D_{3} H(s(v, u, \lambda))\right\| d s<E .
$$

By means of $\bar{\varepsilon}$ we further define

$$
\bar{c}(K):=\frac{\bar{\varepsilon}(K)}{2 \cdot 7 K^{2} C^{2} E}, \quad \tilde{c}:=\frac{5 \bar{\varepsilon}(K)}{7 K C} .
$$

Therefore, we find for $\|v\|<\bar{\varepsilon},|\lambda|,|u|<\bar{c}$ and $K>1$ (recall $C \geq 1$ )

$$
\|H(v, u, \lambda)\| \leq \frac{2 \bar{\varepsilon}}{7 K^{2} C^{2}} \leq \frac{2 \bar{\varepsilon}}{7 K C} .
$$

Finally, estimate (19) yields that for all $|\lambda|,|u|<\bar{c}$ and $\|a\|<\tilde{c}$ the mapping $F(\cdot, a, u, \lambda)$ leaves the closed ball $B(0, \bar{\varepsilon}) \subset V_{\boldsymbol{\omega}}$ invariant.

Moreover, due to the linear dependence of $\hat{v}$ on $(H, a)$ and the estimates (9) and (22), we have

$\left\|D_{1} F(v, a, u, \lambda)\right\| \leq\left\|D_{1} \hat{v}(H, a, u, \lambda)\right\| \cdot\left\|D_{1} H(v, a, u, \lambda)\right\| \leq \hat{C} \frac{1}{7 K C} \leq \frac{1}{7 K}$.

Thus, $F$ is a contraction on $B(0, \bar{\varepsilon})$ and the existence and uniqueness part of the lemma follows immediately from the Banach fixed point theorem.

Applying the implicit function theorem at a solution point of (18) provides the smooth dependence of $v$ on $(a, u, \lambda)$.

Lemma 2.7. Choose $K>1$ and $(a, u, \lambda)$ in accordance with lemma [2.6. We define functions

$$
\begin{aligned}
& a_{\perp}^{+}(a, u, \lambda):=P_{s}^{+}(u, \lambda)\left(\omega^{+}\right) v^{+}(a, u, \lambda)\left(\omega^{+}\right), \\
& a_{\perp}^{-}(a, u, \lambda):=P_{u}^{-}(u, \lambda)\left(-\omega^{-}\right) v^{-}(a, u, \lambda)\left(-\omega^{-}\right) .
\end{aligned}
$$

There are constants $C, \delta^{s}, \delta^{u}>0$, that do not depend on $K$, such that with $\tilde{c}$ according to 23

$$
\begin{aligned}
& \left|a_{\perp}^{+}(a, u, \lambda)\right| \leq C\|a\| e^{-\delta^{s} \omega^{+}}+\frac{\tilde{c}}{K} \\
& \left|a_{\perp}^{-}(a, u, \lambda)\right| \leq C\|a\| e^{-\delta^{u} \omega^{-}}+\frac{\tilde{c}}{K}
\end{aligned}
$$

and

$$
\begin{aligned}
& \left|D_{1} a_{\perp}^{+}(a, u, \lambda)\right| \leq C e^{-\delta^{s} \omega^{+}}+\frac{1}{K}, \\
& \left|D_{1} a_{\perp}^{-}(a, u, \lambda)\right| \leq C e^{-\delta^{u} \omega^{-}}+\frac{1}{K} .
\end{aligned}
$$

Proof. From (14) and the estimates in the proof of lemma 2.6 we get

$$
\begin{aligned}
\left|a_{\perp}^{+}(a, u, \lambda)\right| & \leq C\left(\|a\| e^{-\delta^{s} \omega^{+}}+\|H(v, u, \lambda)\|\right) \\
& \leq C\|a\| e^{-\delta^{s} \omega^{+}}+\frac{\tilde{c}}{K}
\end{aligned}
$$


Next we estimate $D_{1} a_{\perp}^{+}(a, u, \lambda)=\frac{\partial}{\partial a} \hat{a}_{\perp}^{+}(H(v(a, u, \lambda), u, \lambda), a, u, \lambda)$. Hence

$$
\begin{gathered}
\left|D_{1} a_{\perp}^{+}(a, u, \lambda)\right| \leq\left|D_{1} \hat{a}_{\perp}^{+}(H, a, u, \lambda)\right| \cdot\left\|D_{1} H(v, u, \lambda)\right\| \cdot\left\|D_{1} v(a, u, \lambda)\right\| \\
+\left|D_{2} \hat{a}_{\perp}^{+}(H, a, u, \lambda)\right| .
\end{gathered}
$$

Because $v(a, u, \lambda)=\hat{v}(H(v(a, u, \lambda), u, \lambda), a, u, \lambda)$ we get

$$
\left\|D_{1} v(a, u, \lambda)\right\| \leq \frac{\left\|D_{2} \hat{v}(H, a, u, \lambda)\right\|}{1-\left\|D_{1} \hat{v}(H, a, u, \lambda)\right\| \cdot\left\|D_{1} H(v, u, \lambda)\right\|} .
$$

Recall that $\left\|D_{2} \hat{v}(H, a, u, \lambda)\right\| \leq C,\left\|D_{1} \hat{v}(H, a, u, \lambda)\right\| \leq C$ and $\left\|D_{1} H(v, u, \lambda)\right\|<\frac{1}{7 K C}$, hence

$$
\left\|D_{1} v(a, u, \lambda)\right\| \leq \frac{7 K C}{7 K-1} .
$$

Together with (15) and (16) we finally get

$$
\left|D_{1} a_{\perp}^{+}(a, u, \lambda)\right| \leq 2 C \frac{1}{7 K C^{2}} \frac{7 K C}{7 K-1}+C e^{-\delta^{s} \omega^{+}} \leq \frac{1}{K}+C e^{-\delta^{s} \omega^{+}} .
$$

With similar computations for $a_{\perp}^{-}$, the estimates of the lemma follow.

Recapitulating, we want to note that we find solutions according to theorem 2.2 by inserting the solutions $v(a, u, \lambda)$ into the representation (6).

The statement of theorem 2.2 remains true for ' $\omega^{-}=\infty$ ' in the following sense:

Corollary 2.8. Fix $\omega^{+}$. There is a constant $c>0$ such that for $|\lambda|<c, u \in U,|u|<c$, and $a^{+} \in \mathbb{R}^{n},\left|a^{+}\right|<c$, there is a unique pair of solutions $\left(x^{-}, x^{+}\right)$of (1) that satisfy

$(J) x^{-/+}\left(a^{+}, u, \lambda\right)(0) \in \Sigma, x^{-}\left(a^{+}, u, \lambda\right)(0) \in W_{\lambda}^{u}\left(\gamma^{-}\right)$,

$x^{-}\left(a^{+}, u, \lambda\right)(0)-x^{+}\left(a^{+}, u, \lambda\right)(0) \in Z$ and

$\left(B^{+}\right)\left(i d-P^{+}(u, \lambda)\left(\omega^{+}\right)\right)\left(x^{+}\left(a^{+}, u, \lambda\right)\left(\omega^{+}\right)-q^{+}(u, \lambda)\left(\omega^{+}\right)-a^{+}\right)=0$.

Proof. Basically the statement follows by setting (id $\left.-P^{-}(u, \lambda)\left(-\omega^{-}\right)\right) a^{-}=0$ in theorem 2.2: Let $\omega^{-}$be any value in accordance with theorem 2.2. Then, due to $\left(\mathrm{id}-P^{-}(u, \lambda)\left(-\omega^{-}\right)\right) a^{-}=0$, it follows that $\hat{v}^{-}\left(-\omega^{-}\right) \in \operatorname{im} P^{-}(u, \lambda)\left(-\omega^{-}\right)=$ $T_{q^{-}(u, \lambda)\left(-\omega^{-}\right)} W_{\lambda}^{u}\left(\gamma^{-}\right)$, cf. Lemma 2.4. Assuming that $W_{\lambda}^{u}\left(\gamma^{-}\right)$is flat around $q^{-}(u, \lambda)\left(-\omega^{-}\right)$, meaning that locally around $q^{-}(u, \lambda)\left(-\omega^{-}\right)$the unstable manifold $W_{\lambda}^{u}\left(\gamma^{-}\right)$and $q^{-}(u, \lambda)\left(-\omega^{-}\right)+T_{q^{-}(u, \lambda)\left(-\omega^{-}\right)} W_{\lambda}^{u}\left(\gamma^{-}\right)$coincide, we find $q^{-}(u, \lambda)\left(-\omega^{-}\right)+$ $\hat{v}^{-}\left(-\omega^{-}\right) \in W_{\lambda}^{u}\left(\gamma^{-}\right)$.

Solving fixed point equation (18) with that particular $\hat{v}$, we find that $x^{-}(u, \lambda)\left(-\omega^{-}\right)=q^{-}(u, \lambda)\left(-\omega^{-}\right)+v^{-}\left(-\omega^{-}\right) \in W_{\lambda}^{u}\left(\gamma^{-}\right)$; compare also (6) . Hence $x^{-}$lies in the unstable manifold of $\gamma^{-}$.

Remark 2.9. In the same sense theorem 2.2 remains true for ' $\omega^{+}=\infty$ '. 


\subsection{The jump function for a short Lin orbit segment}

According to theorem 2.2, for given $a=\left(a^{-}, a^{+}\right), u, \lambda$ and $\omega=\left(\omega^{-}, \omega^{+}\right)$, there is a unique short Lin orbit segment $X=\left(x^{-}, x^{+}\right)$. Note that $X$ depends in particular on $\boldsymbol{\omega}$, which is not reflected in our notation so far. To emphasize this dependence from we now use the notation $X_{\boldsymbol{\omega}}$, and similarly $x_{\boldsymbol{\omega}}^{ \pm}$and $v_{\boldsymbol{\omega}}^{ \pm}$. We define the jump function $\Xi$ as

$$
\Xi(\boldsymbol{\omega}, a, u, \lambda):=x_{\boldsymbol{\omega}}^{-}(a, u, \lambda)(0)-x_{\boldsymbol{\omega}}^{+}(a, u, \lambda)(0) .
$$

Using that $x_{\boldsymbol{\omega}}^{-/+}(a, u, \lambda)(t)=q^{-/+}(u, \lambda)(t)+v_{\boldsymbol{\omega}}^{-/+}(a, u, \lambda)(t)$, we can write $\Xi$ in the form

$$
\Xi(\boldsymbol{\omega}, a, u, \lambda)=\xi^{\infty}(u, \lambda)+\xi(\boldsymbol{\omega}, a, u, \lambda),
$$

where

$$
\begin{aligned}
& \xi^{\infty}(u, \lambda):=q^{-}(u, \lambda)(0)-q^{+}(u, \lambda)(0), \\
& \xi(\boldsymbol{\omega}, a, u, \lambda):=v_{\boldsymbol{\omega}}^{-}(a, u, \lambda)(0)-v_{\boldsymbol{\omega}}^{+}(a, u, \lambda)(0) .
\end{aligned}
$$

Recall that $\left(v_{\boldsymbol{\omega}}^{-}, v_{\boldsymbol{\omega}}^{+}\right)$is the solution of the fixed point equation (18) and, hence, solves the boundary value problem $\left((\mathbb{7}),\left(J_{v}\right),\left(B_{v}^{-}\right),\left(B_{v}^{+}\right)\right)$.

The term $\xi^{\infty}$ reflects the intersection of the stable and unstable manifolds of $\gamma^{-}$ and $\gamma^{+}$respectively. We present examples for suitable choices of $\xi^{\infty}$ in section 4 . Here we focus on estimates of $\xi$.

In order to establish those estimates, we impose some assumptions on the leading eigenvalues of $\gamma^{-}$and $\gamma^{+}$. Let $\mu_{s}^{-}$denote the leading stable eigenvalue or the leading stable Floquet exponent of $\gamma^{-}$depending on whether $\gamma^{-}$is an equilibrium point or a periodic orbit with minimal period $T^{-}>0$. Similarly, let $\mu_{u}^{+}$be the leading unstable eigenvalue or the leading unstable Floquet exponent of $\gamma^{+}$. We assume the following:

Hypothesis 2.1. $\mu_{s}^{-}, \mu_{u}^{+}$are real and simple.

Further, for the sake of simplicity, we also assume

Hypothesis 2.2. $\operatorname{dim} Z=1$.

Let $Z=\operatorname{span}\{z\},|z|=1$.Then, since $\xi \in Z$,

$$
\xi(\boldsymbol{\omega}, a, u, \lambda)=\langle z, \xi(\boldsymbol{\omega}, a, u, \lambda)\rangle z .
$$

Further we assume

Hypothesis 2.3. The direct sum decomposition (3) is orthogonal with respect to the used scalar product $\langle\cdot, \cdot\rangle$.

Lemma 2.10. Let $a, u, \lambda, \boldsymbol{\omega}$ be in accordance with theorem 2.2, and let hypotheses 2.12.3 hold. Then,

$$
\xi(\boldsymbol{\omega}, a, u, \lambda)=\mathcal{O}(\|a\|) .
$$

The $\mathcal{O}(\cdot)$ limit holds for $\|a\| \rightarrow 0$ uniformly in $u, \lambda, \boldsymbol{\omega}$. 
Proof. According to the definition of $\xi$ and hypothesis 2.3 we find that

$$
\begin{aligned}
\langle z, \xi(\boldsymbol{\omega}, a, u, \lambda)\rangle= & \left\langle z, v_{\boldsymbol{\omega}}^{-}(a, u, \lambda)(0)-v_{\boldsymbol{\omega}}^{+}(a, u, \lambda)(0)\right\rangle \\
= & \left\langle z,\left(\mathrm{id}-P^{-}(u, \lambda)(0)\right) v_{\boldsymbol{\omega}}^{-}(a, u, \lambda)(0)\right\rangle \\
& \quad-\left\langle z,\left(\mathrm{id}-P^{+}(u, \lambda)(0)\right) v_{\boldsymbol{\omega}}^{+}(a, u, \lambda)(0)\right\rangle .
\end{aligned}
$$

Since $v$ satisfies the fixed point equation (18), according to (11) we find that

$$
\begin{aligned}
\langle z, \xi(\boldsymbol{\omega}, a, u, \lambda)\rangle= & \left\langle\Phi^{-}\left(0,-\omega^{-}\right)^{T} z,\left(\mathrm{id}-P^{-}(u, \lambda)\left(-\omega^{-}\right)\right) a^{-}\right\rangle \\
& -\left\langle\Phi^{+}\left(0, \omega^{+}\right)^{T} z,\left(\mathrm{id}-P^{+}(u, \lambda)\left(\omega^{+}\right)\right) a^{+}\right\rangle \\
& +\left\langle z, \int_{-\omega^{-}}^{0} \Phi^{-}(0, \tau)\left(\mathrm{id}-P^{-}(\tau)\right) h^{-}\left(\tau, v_{\boldsymbol{\omega}}^{-}(\tau), u, \lambda\right) d \tau\right\rangle \\
& +\left\langle z, \int_{0}^{\omega^{+}} \Phi^{+}(0, \tau)\left(\mathrm{id}-P^{+}(\tau)\right) h^{+}\left(\tau, v_{\boldsymbol{\omega}}^{+}(\tau), u, \lambda\right) d \tau\right\rangle .
\end{aligned}
$$

First note that

$$
\left\langle\Phi^{+}\left(0, \omega^{+}\right)^{T} z,\left(\mathrm{id}-P^{+}(u, \lambda)\left(\omega^{+}\right)\right) a^{+}\right\rangle=\left\langle\Phi^{+}\left(0, \omega^{+}\right)^{T}\left(\mathrm{id}-P^{+}(0)\right)^{T} z, a^{+}\right\rangle .
$$

Further, $\Phi^{+}(0, \cdot)^{T}$ is a solution of the adjoint of the variational equation along $\gamma^{+}$. Exponential dichotomy/trichotomy of this equation yields that, uniformly in $u, \lambda, \omega^{+}$,

$$
\left\langle\Phi^{+}\left(0, \omega^{+}\right)^{T}\left(\mathrm{id}-P^{+}(0)\right)^{T} z, a^{+}\right\rangle=\mathcal{O}\left(\left|a^{+}\right|\right) .
$$

Similar arguments apply to $\left\langle\Phi^{-}\left(0,-\omega^{-}\right)^{T} z,\left(\right.\right.$ id $\left.\left.-P^{-}(u, \lambda)\left(-\omega^{-}\right)\right) a^{-}\right\rangle$.

Standard results from Lin's method (cf. [10, 18, 19]) imply that the integral terms in (29) are also $\mathcal{O}\left(\left|a^{-}\right|\right)$or $\mathcal{O}\left(\left|a^{+}\right|\right)$uniformly in $u, \lambda, \boldsymbol{\omega}$, respectively. Note that the arguments in [10, 19], where $\gamma^{ \pm}$are always equilibria, apply also in the present situation. These arguments are mainly based on the exponential dichotomy of the variational equation along $\gamma^{ \pm}$and the structure of $h$.

Corollary 2.11. Let $a^{+}, u, \lambda, \omega^{+}$be in accordance with corollary 2.8, and let hypotheses 2.12 .3 hold. Then,

$\left\langle z, \xi\left(\omega^{+}, a^{+}, u, \lambda\right)\right\rangle=-\left\langle\Phi^{+}\left(0, \omega^{+}\right)^{T} z,\left(i d-P^{+}(u, \lambda)\left(\omega^{+}\right)\right) a^{+}\right\rangle+o\left(\left|a^{+}\right|\right)$.

The o $(\cdot)$ limit holds for $\left|a^{+}\right| \rightarrow 0$ uniformly in $u, \lambda, \omega^{+}$.

Proof. As in the proof of corollary 2.8, we set $a^{-}=0$. Then estimates in [19, lemma 3.20] provide the the corresponding estimate of the integral terms in (29).

\section{Joining two short Lin orbit segments}

Let $\gamma_{l} \cup q_{l} \cup \gamma$ and $\gamma \cup q_{r} \cup \gamma_{r}$ be consecutive short heteroclinic chain segments. The objective of this section is to join the related short Lin orbit segments $X_{l}=\left(x_{l}^{-}, x_{l}^{+}\right)$ and $X_{r}=\left(x_{r}^{-}, x_{r}^{+}\right)$to a long Lin orbit segment. Here we focus on the case where $\gamma$ is a hyperbolic periodic orbit with minimal period $T>0$. We use the same notation as in section 2 with an additional subscript ' $l$ ' or ' $r$ ' referring to the left short Lin orbit 
segments $X_{l}$ or right short Lin orbit segments $X_{r}$, respectively. However, for convenience we use the short notation $\omega^{-}=\omega_{l}^{-}$and $\omega^{+}=\omega_{r}^{+}$.

In the construction the transition time $\tau$ from $\Sigma_{l}$ to $\Sigma_{r}$ plays a major role. In the present context, $\tau$ is directly related to the number $\nu$ of rotations of the long Lin orbit segment along $\gamma$.

Theorem 3.1. Fix $\omega^{-}, \omega^{+}>0$. There are constants $c, N>0$ such that for all $|\lambda|<c$, $u=\left(u_{l}, u_{r}\right),\|u\|<c, a^{-}, a^{+} \in \mathbb{R}^{n},\left|a_{l}^{-}\right|,\left|a_{r}^{+}\right|<c$, and for all $\nu \in \mathbb{N} \cap(N, \infty)$, there is a transition time $\tau$ and a unique triple $x=\left(x_{l}, x_{m}, x_{r}\right), x(\cdot)=x\left(\nu, a_{l}^{-}, a_{r}^{+}, u, \lambda\right)(\cdot)$, of solutions of (1) that satisfy

$(J) x_{l}(0), x_{m}(0) \in \Sigma_{l}, x_{m}(\tau), x_{r}(0) \in \Sigma_{r}, x_{l}(0)-x_{m}(0) \in Z_{l}, x_{m}(\tau)-x_{r}(0) \in Z_{r}$,

$\left(B_{l}\right)\left(i d-P_{l}^{-}\left(u_{l}, \lambda\right)\left(-\omega^{-}\right)\right)\left(x_{l}\left(-\omega^{-}\right)-q_{l}^{-}\left(u_{l}, \lambda\right)\left(-\omega^{-}\right)-a^{-}\right)=0$,

$\left(B_{r}\right)\left(i d-P_{r}^{+}\left(u_{r}, \lambda\right)\left(\omega^{+}\right)\right)\left(x_{r}\left(\omega^{+}\right)-q_{r}^{+}\left(u_{r}, \lambda\right)\left(\omega^{+}\right)-a^{+}\right)=0$.

Figure 4 visualizes the statement of the theorem.

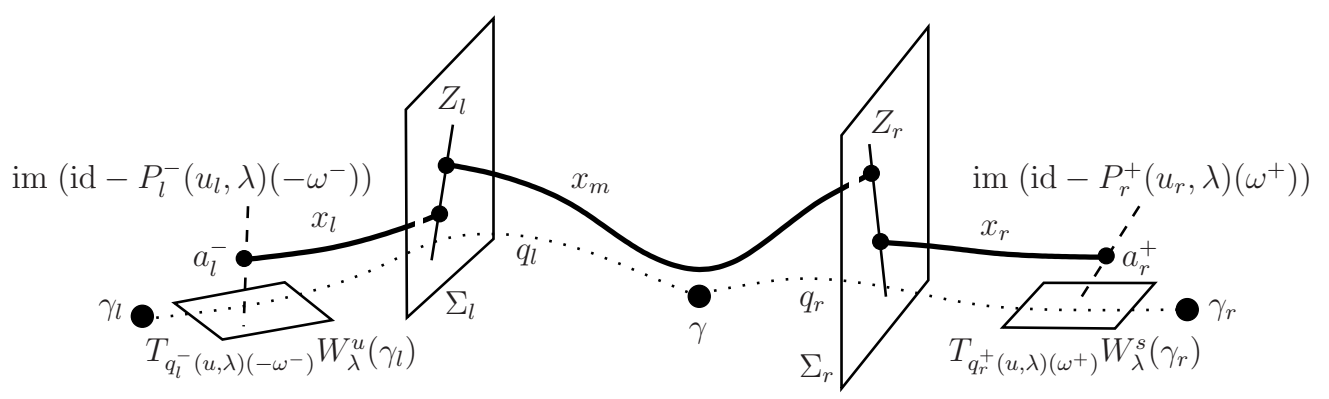

Figure 4. Sketch of a long Lin orbit segment $\left(x_{l}, x_{m}, x_{r}\right)$ near a long heteroclinic chain $\gamma_{l} \cup q_{l} \cup \gamma \cup q_{r} \cup \gamma_{r}$.

We perform the proof of theorem 3.1 in two steps, see sections 3.1 and 3.2 . First we study the flow along $\gamma$ by means of a Poincaré map $\Pi: \Sigma_{\gamma} \rightarrow \Sigma_{\gamma}$, where $\Sigma_{\gamma}$ is an appropriate Poincaré section. More precisely, we show that there are П-orbit segments $y$ satisfying certain boundary conditions in $\Sigma_{\gamma}$. To that end, we employ a similar technique as used in the theory of Lin's method for discrete dynamical systems [10]. We denote the $f$-orbit that is the suspension of the $\Pi$-orbit $y$ by $x(y)$. Then we couple $x_{l}^{+}$and $x(y)$ and simultaneously $x(y)$ and $x_{r}^{-}$. The partial orbit $x_{m}$ is composed of $x_{l}^{+}, x(y)$ and $x_{r}^{-}$. The (in this context prescribed) times $\omega_{l}^{+}$and $\omega_{r}^{-}$and the duration of $x(y)$ add up to the transition time $\tau$. Further, we have $x_{l}=x_{l}^{-}$and $x_{r}=x_{r}^{+}$.

The statement of theorem 3.1 remains true for $\omega^{-}=\omega^{+}=\infty$ in the following sense:

Corollary 3.2. There are constants $c, N>0$ such that for all $|\lambda|<c, u=\left(u_{l}, u_{r}\right)$, $\left|u_{l}\right|,\left|u_{r}\right|<c$, and for all $\nu \in \mathbb{N} \cap(N, \infty)$, there is a unique triple $x=\left(x_{l}, x_{m}, x_{r}\right)$, $x(\cdot)=x(\nu, u, \lambda)(\cdot)$, of solutions of (11) such that for some transition time $\tau$ $(J) x_{l}(0), x_{m}(0) \in \Sigma_{l}, x_{m}(\tau), x_{r}(0) \in \Sigma_{r}, x_{l}(0)-x_{m}(0) \in Z_{l}, x_{m}(\tau)-x_{r}(0) \in Z_{r}$, 
(B) $x_{l}(0) \in W^{u}\left(\gamma_{l}\right), \quad x_{r}(0) \in W^{s}\left(\gamma_{r}\right)$.

For fixed $\nu$ the solution $x$ depends smoothly on $(u, \lambda)$.

The proof of corollary 3.2 will be given at the end of section 3.2 .

Definition 3.3. If $\omega^{-}, \omega^{+}<\infty$ we call the triple $\left(x_{l}, x_{m}, x_{r}\right)$ a long Lin orbit segment, and in case $\omega^{-}=\omega^{+}=\infty$ we call $\left(x_{l}, x_{m}, x_{r}\right)$ a heteroclinic Lin orbit connecting $\gamma_{l}$ and $\gamma_{r}$. If $\gamma_{l} \equiv \gamma_{r}$, we call $\left(x_{l}, x_{m}, x_{r}\right)$ a homoclinic Lin orbit connecting $\gamma_{l}$ to itself.

\subsection{The flow near $\gamma$}

Let $\Sigma_{\gamma}$ be a Poincaré section of $\gamma$. We consider the discrete dynamical system defined by the Poincaré map $\Pi: \Sigma_{\gamma} \times \mathbb{R}^{m} \rightarrow \Sigma_{\gamma}$ near $p_{\gamma}:=\gamma \cap \Sigma_{\gamma}$ :

$$
y(n+1)=\Pi(y(n), \lambda) .
$$

The intersection points of $q_{l}^{+}\left(u_{l}, \lambda\right)$ and $q_{r}^{-}\left(u_{r}, \lambda\right)$ with the Poincaré section $\Sigma_{\gamma}$ define solutions $q_{d}^{+}\left(u_{l}, \lambda\right)(n), q_{d}^{-}\left(u_{r}, \lambda\right)(n)$ of (30) lying in the stable/unstable manifold of the hyperbolic П-equilibrium $p_{\gamma}$. Let $Y_{\gamma}$ be the $(n-1)$-dimensional subspace of $\mathbb{R}^{n}$ such that

$$
\Sigma_{\gamma}=p_{\gamma}+Y_{\gamma}
$$

The variational equation along $q_{d}^{ \pm}$has an exponential dichotomy on $\mathbb{Z}^{ \pm}$and we denote the corresponding projections by $Q^{+}\left(u_{l}, \lambda\right)$ and $Q^{-}\left(u_{r}, \lambda\right)$. Note again that the images of $Q^{ \pm}$are well-determined:

$$
\begin{aligned}
& \operatorname{im} Q^{+}\left(u_{l}, \lambda\right)(0)=T_{q_{d}^{+}\left(u_{l}, \lambda\right)(0)} W_{\Pi, \lambda}^{s}\left(p_{\gamma}\right) \subset T_{q_{d}^{+}\left(u_{l}, \lambda\right)(0)} W_{\lambda}^{s}(\gamma), \\
& \operatorname{im} Q^{-}\left(u_{r}, \lambda\right)(0)=T_{q_{d}^{-}\left(u_{r}, \lambda\right)(0)} W_{\Pi, \lambda}^{u}\left(p_{\gamma}\right) \subset T_{q_{d}^{-}\left(u_{r}, \lambda\right)(0)} W_{\lambda}^{u}(\gamma),
\end{aligned}
$$

where $W_{\Pi, \lambda}^{s(u)}$ denotes the (un)stable manifold of the mapping $\Pi=\Pi(\lambda)$ and we use the short notation $W_{\Pi}^{s(u)}$ at $\lambda=0$. However, there is some freedom in choosing the kernels of $Q^{ \pm}$, which allows us to use the ideas from Lin's method for discrete dynamical systems in the following, and also allows us to couple the solution of the discrete system with the solutions of the continuous system, cf. (49) and (50) below.

Lemma 3.4. There are constants $\tilde{c}, \bar{c}>0$ and $N \in \mathbb{N}$ such that for all $|\lambda|<\bar{c}$, $u=\left(u_{l}, u_{r}\right), u_{l} \in U_{l}, u_{r} \in U_{r},\left|u_{l}\right|,\left|u_{r}\right|<\bar{c}, \nu>N$ and $b=\left(b^{+}, b^{-}\right) \in Y_{\gamma} \times Y_{\gamma},\|b\|<\tilde{c}$, there is a unique solution $y=y\left(b, u_{l}, u_{r}, \lambda\right)$ of (30) that satisfies

$(\mathcal{B}) Q^{+}\left(u_{l}, \lambda\right)(0)\left(y(b, u, \lambda)(0)-q_{d}^{+}\left(u_{l}, \lambda\right)(0)-b^{+}\right)=0$

$Q^{-}\left(u_{r}, \lambda\right)(0)\left(y(b, u, \lambda)(\nu)-q_{d}^{-}\left(u_{r}, \lambda\right)(0)-b^{-}\right)=0$.

Again we suppress the dependence of $y$ on $\nu$ from our notation. Note that this lemma is a discrete version of the existence and uniqueness result on Shilnikov data, cf. [8]. However, also in view of its application in the following section [3.2, we consider a reformulation by using small perturbations of $q_{d}^{+}(n)$ and $q_{d}^{-}(n)$, similar to section 2 . For 
Heteroclinic chains involving periodic orbits

given $\nu \in \mathbb{N}$ we define $\nu^{+}:=\left\lfloor\frac{\nu}{2}\right\rfloor$, the integer part of $\nu$, and $\nu^{-}:=\nu-\nu^{+}$. Further, let us think of $y$ as being composed of two partial orbits as follows

$$
y(n)= \begin{cases}y^{+}(n), & n \in\left[0, \nu^{+}\right] \cap \mathbb{N} \\ y^{-}(n-\nu), & n \in\left[\nu^{+}, \nu\right] \cap \mathbb{N}\end{cases}
$$

with the additional demand that

$$
y^{+}\left(\nu^{+}\right)=y^{-}\left(-\nu^{-}\right) .
$$

We write

$$
y^{+}(n)=q_{d}^{+}\left(u_{l}, \lambda\right)(n)+w^{+}(n) \text { and } y^{-}(n)=q_{d}^{-}\left(u_{r}, \lambda\right)(n)+w^{-}(n) .
$$

If $y^{ \pm}$solve (30), then $w^{ \pm}(\cdot)$ satisfy the following difference equations:

$$
\begin{aligned}
& w^{-}(n+1)=D_{1} \Pi\left(q_{d}^{-}\left(u_{r}, \lambda\right)(n), \lambda\right) w^{-}(n)+h^{-}\left(n, w^{-}, u_{r}, \lambda\right), \\
& w^{+}(n+1)=D_{1} \Pi\left(q_{d}^{+}\left(u_{l}, \lambda\right)(n), \lambda\right) w^{+}(n)+h^{+}\left(n, w^{+}, u_{l}, \lambda\right),
\end{aligned}
$$

where

$$
\begin{gathered}
h^{ \pm}(n, w, u, \lambda):=\Pi\left(q_{d}^{ \pm}(u, \lambda)(n)+w, \lambda\right)-\Pi\left(q_{d}^{ \pm}(u, \lambda)(n), \lambda\right) \\
-D_{1} \Pi\left(q_{d}^{ \pm}(u, \lambda)(n), \lambda\right) w .
\end{gathered}
$$

For $\nu \in \mathbb{N}$ let $S_{\nu}$ denote the space of functions $\{0, \ldots, \nu\} \rightarrow Y_{\gamma}$, and let $S_{-\nu}$ denote the space of functions $\{-\nu, \ldots, 0\} \rightarrow Y_{\gamma}$. For given $\nu^{+}$and $\nu^{-}$we write $\boldsymbol{\nu}:=\left(\nu^{+}, \nu^{-}\right)$, and we define the space

$$
W_{\nu}:=S_{\nu^{+}} \times S_{-\nu^{-}} .
$$

Then lemma 3.4 follows from

Lemma 3.5. There are constants $\bar{\epsilon}, \tilde{\mathfrak{c}}, \overline{\mathfrak{c}}$ and $N \in \mathbb{N}$ such that for $|\lambda| \leq \overline{\mathfrak{c}}, u:=\left(u_{l}, u_{r}\right) \in$ $U_{l} \times U_{r}$, with $\|u\| \leq \overline{\mathfrak{c}}, \nu>N$ and $b=\left(b^{+}, b^{-}\right) \in Y_{\gamma} \times Y_{\gamma},\|b\| \leq \tilde{\mathfrak{c}}$, there is a unique pair $w_{\boldsymbol{\nu}}=\left(w_{\boldsymbol{\nu}}^{+}, w_{\boldsymbol{\nu}}^{-}\right) \in W_{\boldsymbol{\nu}}, w_{\boldsymbol{\nu}}=w_{\boldsymbol{\nu}}(b, u, \lambda)$, of solutions of (35) in an $\bar{\epsilon}$-neighborhood of $0 \in W_{\nu}$ such that

$\left(\mathcal{B}_{w}\right) Q^{+}\left(u_{l}, \lambda\right)(0)\left(w_{\nu}^{+}(b, u, \lambda)(0)-b^{+}\right)=0, \quad Q^{-}\left(u_{r}, \lambda\right)(0)\left(w_{\nu}^{-}(b, u, \lambda)(0)-b^{-}\right)=0$,

(C) $w_{\nu}^{-}(b, u, \lambda)\left(-\nu^{-}\right)-w_{\nu}^{+}(b, u, \lambda)\left(\nu^{+}\right)=q_{d}^{+}\left(u_{l}, \lambda\right)\left(\nu^{+}\right)-q_{d}^{-}\left(u_{r}, \lambda\right)\left(-\nu^{-}\right)$.

Proof. To some extent the arguments run parallel to those used in section 2.2. Here we only give a sketch of the proof; for more details we refer to [18].

First we consider the inhomogeneous equations

$$
\begin{aligned}
& w^{-}(n+1)=D_{1} \Pi\left(q_{d}^{-}\left(u_{r}, \lambda\right)(n), \lambda\right) w^{-}(n)+g^{-}(n), \\
& w^{+}(n+1)=D_{1} \Pi\left(q_{d}^{+}\left(u_{l}, \lambda\right)(n), \lambda\right) w^{+}(n)+g^{+}(n),
\end{aligned}
$$

with boundary conditions

$$
\begin{aligned}
& \left(\mathcal{B}_{w}\right) Q^{+}\left(u_{l}, \lambda\right)(0)\left(w^{+}(0)-b^{+}\right)=0, \quad Q^{-}\left(u_{r}, \lambda\right)(0)\left(w^{-}(0)-b^{-}\right)=0, \\
& \left(\mathcal{B}_{\beta}\right)\left(\mathrm{id}-Q^{+}\left(u_{l}, \lambda\right)\left(\nu^{+}\right)\right) w^{+}\left(\nu^{+}\right)=\beta^{+}, \quad\left(\mathrm{id}-Q^{-}\left(u_{r}, \lambda\right)\left(-\nu^{-}\right)\right) w^{-}\left(-\nu^{-}\right)=\beta^{-}
\end{aligned}
$$


for given $\beta^{+} \in \operatorname{im}\left(\mathrm{id}-Q^{+}\left(u_{l}, \lambda\right)\left(\nu^{+}\right)\right)$and $\beta^{-} \in \operatorname{im}\left(\mathrm{id}-Q^{-}\left(u_{r}, \lambda\right)\left(-\nu^{-}\right)\right)$. We write $\beta:=\left(\beta^{+}, \beta^{-}\right)$. Similar to the proof of lemma 2.4, we find that the boundary value problem $\left((\underline{36}),\left(B_{b}\right),\left(B_{\beta}\right)\right)$ has a unique solution $\bar{w} \in W_{\nu}, \bar{w}=\bar{w}(g, b, \beta, u, \lambda)$.

Next we replace the boundary condition $\left(\mathcal{B}_{\beta}\right)$ by

$\left(\mathcal{B}_{d}\right) w^{+}\left(\nu^{+}\right)-w^{-}\left(-\nu^{-}\right)=d, \quad d \in Y_{\gamma}$.

Indeed there is a $\beta=\beta(d)$ such that $\hat{w}(g, b, d, u, \lambda):=\bar{w}(g, b, \beta(d), u, \lambda)$ is the unique solution of the boundary value problem $\left(\underline{(36)},\left(B_{b}\right),\left(B_{d}\right)\right)$. The argument for this fact runs parallel to the corresponding construction in [19] or [10].

Further, similar to the proof of lemma 2.6, we consider a fixed point equation whose solutions also satisfy the coupling condition $(\mathcal{C})$ : For that we define

$$
d_{\boldsymbol{\nu}}(u, \lambda):=q_{d}^{+}\left(u_{l}, \lambda\right)\left(\nu^{+}\right)-q_{d}^{-}\left(u_{r}, \lambda\right)\left(-\nu^{-}\right) .
$$

Finally, we consider the fixed point equation

$$
w=\hat{w}\left(\mathcal{H}(w, u, \lambda), b, d_{\boldsymbol{\nu}}, u, \lambda\right) .
$$

Here, $\mathcal{H}$ is the discrete pendant of the Nemytskii operator defined in (17). Similar to the procedure in section 2.2 one proves that (38) has a unique fixed point.

We define functions $B_{Y_{\gamma} \times Y_{\gamma}}(0, \tilde{\mathfrak{c}}) \times B_{U_{l} \times U_{r}}(0, \overline{\mathfrak{c}}) \times B_{\mathbb{R}^{m}}(0, \overline{\mathfrak{c}}) \rightarrow B_{Y_{\gamma}}(0, \bar{\epsilon})$

$$
\begin{aligned}
& b_{\perp}^{+}(b, u, \lambda):=\left(\mathrm{id}-Q^{+}\left(u_{l}, \lambda\right)(0)\right) w_{\nu}^{+}(b, u, \lambda)(0), \\
& b_{\perp}^{-}(b, u, \lambda):=\left(\mathrm{id}-Q^{-}\left(u_{r}, \lambda\right)(0)\right) w_{\nu}^{-}(b, u, \lambda)(0) .
\end{aligned}
$$

First note that $b_{\perp}^{ \pm}$depend smoothly on $(b, u, \lambda)$, and note further that $b_{\perp}^{ \pm}$depend also on $\boldsymbol{\nu}$. The 'size' of these functions is closely related to the 'size' of the jumps $\xi_{l}$ and $\xi_{r}$.

In accordance with hypothesis 2.1 we assume

Hypothesis 3.1. The leading stable and unstable eigenvalues $\mu^{s / u}$ of $p_{\gamma}$ are real and simple.

Note that here $\mu^{s / u}$ denote the eigenvalues of $p_{\gamma}$ (and not the Floquet exponents of $\gamma$ as in section 2).

Hypothesis 3.2. $q_{d}^{+}\left(u_{l}, \lambda\right)(\cdot)$ and $q_{d}^{-}\left(u_{r}, \lambda\right)(\cdot)$ approach $p_{\gamma}$ along the leading stable and unstable direction, respectively.

Hypothesis 3.3. $b_{\perp}^{+}(b, u, \lambda)$ and $b_{\perp}^{-}(b, u, \lambda)$ are not in the strong stable subspace of the adjoint of the variational equation along $q^{+}\left(u_{l}, \lambda\right)(\cdot)$ and $q^{-}\left(u_{r}, \lambda\right)(\cdot)$, respectively, for $n=0$.

Lemma 3.6. Assume hypotheses 3.1] 3.3. Further, let the assumptions of lemma 3.5 hold. There are functions $c^{s / u}=c^{s / u}(b, u, \lambda)$ such that

$$
\begin{aligned}
& \left|b_{\perp}^{+}(b, u, \lambda)\right|=c^{u}(b, u, \lambda)\left(\mu^{u}\right)^{-\nu}+o\left(\left|\mu^{u}\right|^{-\nu}\right), \\
& \left|b_{\perp}^{-}(b, u, \lambda)\right|=c^{s}(b, u, \lambda)\left(\mu^{s}\right)^{\nu}+o\left(\left|\mu^{s}\right|^{\nu}\right) .
\end{aligned}
$$

There is a constant $C>0$ such that

$$
\left|D_{1} b_{\perp}^{+}(b, u, \lambda)\right| \leq C\left|\mu^{u}\right|^{-\nu}, \quad\left|D_{1} b_{\perp}^{-}(b, u, \lambda)\right| \leq C\left|\mu^{s}\right|^{\nu} .
$$


The functions $c^{u / s}$ are smooth and $c^{u / s}(0,0,0) \neq 0$. The o(.)-terms are valid for $\nu$ tending to infinity.

The estimates in [8] (applied to discrete systems) already provide that $\left|b_{\perp}^{+}(b, u, \lambda)\right|=$ $\mathcal{O}\left(\left|\mu^{u}\right|^{-\nu}\right)$, cf. also corollary [3.7, but they do not give information about the leading term. However, this information is important for the jump estimates and consequently for the construction of bifurcation equations. Note that the information about leading terms of the derivatives in (41) is not needed for our purposes here, but can be computed in a similar manner as in [10, 19].

Proof. With $\hat{b}_{\perp}^{+}=\hat{b}_{\perp}^{+}(b, u, \lambda):=b_{\perp}^{+}(b, u, \lambda) /\left|b_{\perp}^{+}(b, u, \lambda)\right|$ we can write

$$
\left|b_{\perp}^{+}(b, u, \lambda)\right|=\left\langle\hat{b}_{\perp}^{+}, b_{\perp}^{+}(b, u, \lambda)\right\rangle .
$$

Note that $\left(w_{\boldsymbol{\nu}}^{-}, w_{\boldsymbol{\nu}}^{+}\right)$solves (35). So, applying the variation of constants formula to (36) and replacing there $g^{ \pm}$by $h^{ \pm}$finally provides

$$
\begin{aligned}
b_{\perp}^{+}(b, u, \lambda)= & \Psi^{+}\left(0, \nu^{+}\right) \beta^{+} \\
& -\underbrace{\sum_{m=1}^{\nu^{+}} \Psi^{+}(0, m)\left(\mathrm{id}-Q^{+}(0)\right) h^{+}\left(m-1, w^{+}(m-1), u_{l}, \lambda\right)}_{=: \mathcal{S}} .
\end{aligned}
$$

Here $\Psi^{+}(\cdot, \cdot)$ is the transition matrix of the homogeneous equation of (36). Note that $\Psi^{+}$depends on $u_{l}$ and $\lambda$. Further, $\beta^{+}$is defined by the boundary condition $\left(\mathcal{B}_{\beta}\right)$.

Replacing $b_{\perp}^{+}$in the scalar product (42) yields

$$
\begin{aligned}
\left\langle\hat{b}_{\perp}^{+}, b_{\perp}^{+}(b, u, \lambda)\right\rangle & =\left\langle\hat{b}_{\perp}^{+}, \Psi^{+}\left(0, \nu^{+}\right) \beta^{+}\right\rangle-\left\langle\hat{b}_{\perp}^{+}, \mathcal{S}\right\rangle \\
& =\left\langle\Psi^{+}\left(0, \nu^{+}\right)^{T}\left(\mathrm{id}-Q^{+}(0)\right)^{T} \hat{b}_{\perp}^{+}, \beta^{+}\right\rangle-\left\langle\hat{b}_{\perp}^{+}, \mathcal{S}\right\rangle .
\end{aligned}
$$

Considerations similar to those in [10, 19] show that the leading-order term of $b_{\perp}^{+}(b, u, \lambda)$ will be determined by $\left\langle\hat{b}_{\perp}^{+}, \Psi^{+}\left(0, \nu^{+}\right) \beta^{+}\right\rangle$or $\left\langle\Psi^{+}\left(0, \nu^{+}\right)^{T}\left(\mathrm{id}-Q^{+}(0)\right)^{T} \hat{b}_{\perp}^{+}, \beta^{+}\right\rangle$, respectively. Note in this respect that, due to the coupling condition $\left(\mathcal{B}_{\beta}\right)$, the quantity $\beta^{+}$depends on $\boldsymbol{\nu}$.

Computations in [10, 19] show that under hypothesis [3.3

$$
\Psi^{+}\left(0, \nu^{+}\right)^{T}\left(\mathrm{id}-Q^{+}(0)\right)^{T} \hat{b}_{\perp}^{+}=\eta^{+}(b, u, \lambda)\left(\mu^{u}\right)^{-\nu^{+}}+o\left(\left|\mu^{u}\right|^{-\nu^{+}}\right),
$$

where $\eta^{+}(b, u, \lambda) \neq 0$ is a certain eigenvector of $\left(D_{1} \Pi\left(p_{\lambda}, \lambda\right)^{-1}\right)^{T}$ belonging to $\left(\mu^{u}\right)^{-1}$.

Next we consider $\beta^{+}$. Combining $\left(\mathcal{B}_{\beta}\right),\left(\mathcal{B}_{d}\right)$ and (37) yields

$$
\begin{aligned}
\beta^{+}-\beta^{-}= & q_{d}^{-}\left(u_{r}, \lambda\right)\left(-\nu^{-}\right)-q_{d}^{+}\left(u_{l}, \lambda\right)\left(\nu^{+}\right) \\
& -Q^{+}\left(\nu^{+}\right) w^{+}\left(\nu^{+}\right)+Q^{-}\left(-\nu^{-}\right) w^{-}\left(-\nu^{-}\right) .
\end{aligned}
$$

We define projections $\tilde{Q}(\nu)=\tilde{Q}(u, \lambda)(\nu)$ by, cf. [10] for their existence,

$$
\operatorname{im} \tilde{Q}(\nu)=\operatorname{im}\left(\mathrm{id}-Q^{+}\left(\nu^{+}\right)\right) \text {and } \operatorname{ker} \tilde{Q}(\nu)=\operatorname{im}\left(\mathrm{id}-Q^{-}\left(-\nu^{-}\right)\right) .
$$


Applying $\tilde{Q}(\nu)$ to (45) yields

$$
\begin{aligned}
\beta^{+}= & \tilde{Q}(\nu)\left(q_{d}^{-}\left(u_{r}, \lambda\right)\left(-\nu^{-}\right)-q_{d}^{+}\left(u_{l}, \lambda\right)\left(\nu^{+}\right)\right) \\
& -\tilde{Q}(\nu) Q^{+}\left(\nu^{+}\right) w^{+}\left(\nu^{+}\right)+\tilde{Q}(\nu) Q^{-}\left(-\nu^{-}\right) w^{-}\left(-\nu^{-}\right) .
\end{aligned}
$$

In [10] it has been shown that the leading-order term of the right-hand side of (46) is determined by the first addend, and estimates given there reveal that under hypothesis 3.2 we have that

$$
\beta^{+}=\eta^{u}(u, \lambda)\left(\mu^{u}\right)^{-\nu^{-}}+o\left(\left|\mu^{u}\right|^{-\nu^{-}}\right),
$$

where $\eta^{u}\left(u_{l}, \lambda\right) \neq 0$ is a certain eigenvector of $D_{1} \Pi\left(p_{\lambda}, \lambda\right)$ belonging to $\mu^{u}$.

Combining (43), (44) and (47) provides

$$
\left\langle\hat{b}_{\perp}^{+}, b_{\perp}^{+}(b, u, \lambda)\right\rangle=\left\langle\eta^{+}(b, u, \lambda), \eta^{u}(u, \lambda)\right\rangle\left(\mu^{u}\right)^{-\nu}+o\left(\left|\mu^{u}\right|^{-\nu^{-}}\right) .
$$

Here we also used that $\left\langle\hat{b}_{\perp}^{+}, \mathcal{S}\right\rangle=o\left(\left|\mu^{u}\right|^{-\nu}\right)$; we refer again to [10, 19] for details of the necessary computations.

Finally, from the definition of $\eta^{+}$and $\eta^{u}$ it follows that

$$
c^{u}(b, u, \lambda):=\left\langle\eta^{+}(b, u, \lambda), \eta^{u}(u, \lambda)\right\rangle \neq 0 .
$$

More precisely, linear algebra shows that $\eta^{+}$and $\eta^{u}$ cannot be orthogonal. Further, due to hypotheses 3.2 and 3.3 both $\eta^{+}$and $\eta^{u}$ are different from zero.

Similar computations yield the statement on $\left|b_{\perp}^{-}\right|$.

The smoothness of $c^{u / s}$ follows from the smoothness of $\eta^{+}$and $\eta^{u}$. The estimates of the derivatives follow immediately from the considerations in [8].

The following is an immediate consequence of lemma 3.6:

Corollary 3.7. Let the assumptions of lemma 3.6 hold. Then there is a constant $C$ such that for all $(b, u, \lambda) \in B_{Y_{\gamma} \times Y_{\gamma}}(0, \tilde{\mathfrak{c}}) \times B_{U_{l} \times U_{r}}(0, \overline{\mathfrak{c}}) \times B_{\mathbb{R}^{m}}(0, \overline{\mathfrak{c}})$

$$
\left|b_{\perp}^{+}(b, u, \lambda)\right|<C\left(\mu^{u}\right)^{-\nu}, \quad\left|b_{\perp}^{-}(b, u, \lambda)\right|<C\left(\mu^{s}\right)^{-\nu}
$$

\subsection{The coupling within $\Sigma_{\gamma}$}

Now we have all the ingredients to couple two pairs of solutions $\left(x_{l}^{-}, x_{l}^{+}\right)$and $\left(x_{r}^{-}, x_{r}^{+}\right)$ of the continuous system with a solution $y$ of the discrete system, effectively combining the solutions $x_{l}^{+}, x_{r}^{-}$and $y$ into one solution.

We fix the times $\omega_{l}^{+}$and $\omega_{r}^{-}$at sufficiently large values by using a fixed Poincaré section $\Sigma_{\gamma}$ and then switch to the discrete dynamical system to describe the dynamics near the periodic orbit. To reflect the nature of this setting we rename $\omega_{l}^{+}$as $\Omega^{+}$and $\omega_{r}^{-}$as $\Omega^{-}$. We choose $\Omega^{ \pm}$such that $q^{+}\left(u_{l}, \lambda\right)\left(\Omega^{+}\right), q^{-}\left(u_{r}, \lambda\right)\left(-\Omega^{-}\right) \in \Sigma_{\gamma}$.

In our analysis we consider $x_{l / r}^{ \pm}$as perturbations of $q_{l / r}^{ \pm}$:

$$
x_{l / r}^{ \pm}=q_{l / r}^{ \pm}\left(u_{l / r}, \lambda\right)+v_{l / r}^{ \pm}\left(u_{l / r}, \lambda\right) .
$$


Further, we represent, as in the previous section, $y$ as a couple of solutions $\left(y^{+}, y^{-}\right)$, cf. (32), (33), which are written in the form (34). The coupling is performed by searching for $x_{l}, x_{r}$ and $y$ such that

$$
x_{l}^{+}\left(\Omega^{+}\right)=y(0), \quad x_{r}^{-}\left(-\Omega^{-}\right)=y(\nu),
$$

or equivalently in terms of the perturbations

(C) $v_{l}^{+}\left(a_{l}, u_{l}, \lambda\right)\left(\Omega^{+}\right)=w^{+}\left(b, u_{l}, \lambda\right)(0), \quad v_{r}^{-}\left(a_{r}, u_{r}, \lambda\right)\left(-\Omega^{-}\right)=w^{-}\left(b, u_{r}, \lambda\right)(0)$.

The orbit $x_{m}$ is the suspension of the orbit $y$ (see theorem 3.1). Note that the transition time $\tau$ is essentially determined by the 'length $\nu$ ' of the orbit $y$.

For the actual coupling analysis inside the Poincaré section $\Sigma_{\gamma}$, we have to impose a technical assumption.

Hypothesis 3.4. All solutions of (11) in a sufficiently small neighborhood of $q_{l}^{+}$have the same transition time $\Omega^{+}$from $\Sigma_{l}$ to $\Sigma_{\gamma}$. Similarly, all solutions in a sufficiently small neighborhood of $q_{r}^{-}$have the same transition time $\Omega^{-}$from $\Sigma_{\gamma}$ to $\Sigma_{r}$.

This can be achieved simultaneously by a scaling of the vector field in a tubular neighborhood along $q_{l}^{+} / q_{r}^{-}$. Note that this scaling does not influence any of the previous results; see [18] for a similar computation.

Hypothesis 3.4 guarantees that the points $q_{l}^{+}\left(u_{l}, \lambda\right)\left(\Omega^{+}\right)+v_{l}^{+}\left(u_{l}, \lambda\right)\left(\Omega^{+}\right)$and $q_{r}^{-}\left(u_{r}, \lambda\right)\left(-\Omega^{-}\right)+v_{r}^{-}\left(u_{r}, \lambda\right)\left(-\Omega^{-}\right)$are both in $\Sigma_{\gamma}$. Further, this hypothesis allows to determine, cf. also (44) and (5):

$$
\begin{aligned}
\operatorname{ker} Q^{+}\left(u_{l}, \lambda\right)(0) & =\Phi_{l}^{+}\left(u_{l}, \lambda\right)\left(\Omega^{+}, 0\right)\left(W_{l}^{-} \oplus Z_{l}\right) \\
& =\operatorname{ker} P^{+}\left(u_{l}, \lambda\right)\left(\Omega^{+}\right), \\
\operatorname{ker} Q^{-}\left(u_{r}, \lambda\right)(0) & =\Phi_{r}^{-}\left(u_{r}, \lambda\right)\left(\Omega^{-}, 0\right)\left(W_{r}^{-} \oplus Z_{r}\right) \\
& =\operatorname{ker} P^{-}\left(u_{r}, \lambda\right)\left(\Omega^{-}\right),
\end{aligned}
$$

and (31) implies

$$
\begin{aligned}
& \operatorname{im} Q^{+}\left(u_{l}, \lambda\right)(0) \subset \operatorname{im} P_{l}^{+}\left(u_{l}, \lambda\right)\left(\Omega^{+}\right), \\
& \operatorname{im} Q^{-}\left(u_{r}, \lambda\right)(0) \subset \operatorname{im} P_{r}^{-}\left(u_{r}, \lambda\right)\left(-\Omega^{-}\right) .
\end{aligned}
$$

An immediate consequence of (49) and (50) is (cf. also the explanations following lemma 2.3):

Lemma 3.8. The restriction $\left.P_{s, l}^{+}\left(u_{l}, \lambda\right)\left(\Omega^{+}\right)\right|_{\operatorname{im} Q^{+}\left(u_{l}, \lambda\right)(0)}$ acts as an isomorphism $\operatorname{im} Q^{+}\left(u_{l}, \lambda\right)(0) \rightarrow \operatorname{im} P_{s, l}^{+}\left(u_{l}, \lambda\right)\left(\Omega^{+}\right)$. Moreover, for all $v^{+} \in Y_{\gamma}$ we have

$$
\begin{aligned}
& P_{s, l}^{+}\left(u_{l}, \lambda\right)\left(\Omega^{+}\right) Q^{+}\left(u_{l}, \lambda\right)(0) v^{+}=P_{s, l}^{+}\left(u_{l}, \lambda\right)\left(\Omega^{+}\right) v^{+}, \\
& \left(i d-Q^{+}\left(u_{l}, \lambda\right)(0)\right) v^{+}=\left(i d-P_{l}^{+}\left(u_{l}, \lambda\right)\left(\Omega^{+}\right)\right) v^{+} .
\end{aligned}
$$

Similarly $\left.P_{u, r}^{-}\left(u_{r}, \lambda\right)\left(-\Omega^{-}\right)\right|_{\operatorname{im} Q^{-}\left(u_{r}, \lambda\right)(0)}: \operatorname{im} Q^{-}\left(u_{r}, \lambda\right)(0) \rightarrow \operatorname{im} P_{u, r}^{-}(u, \lambda)\left(\Omega^{-}\right)$is an isomorphism, and for all $v^{-} \in Y_{\gamma}$ we have

$$
\begin{aligned}
& P_{u, r}^{-}\left(u_{r}, \lambda\right)\left(-\Omega^{-}\right) Q^{-}\left(u_{r}, \lambda\right)(0) v^{-}=P_{u, r}^{-}\left(u_{r}, \lambda\right)\left(-\Omega^{-}\right) v^{-}, \\
& \left(i d-Q^{-}\left(u_{r}, \lambda\right)(0)\right) v^{-}=\left(i d-P_{r}^{-}\left(u_{r}, \lambda\right)\left(-\Omega^{-}\right)\right) v^{-} .
\end{aligned}
$$


Further, we use the notation

$$
\mathcal{U}:=U_{l} \times U_{r}, \quad \mathcal{U} \ni u=\left(u_{l}, u_{r}\right) .
$$

The following lemma is a reformulation of theorem 3.1 in terms of the perturbances $v_{l}^{ \pm}$, $v_{r}^{ \pm}$and $w^{ \pm}$.

Lemma 3.9. Fix $\omega^{+}, \omega^{-}>0$. There are constants $\Omega^{-}, \Omega^{+}>0, N \in \mathbb{N}, c>0$ such that for all $|\lambda|<c, u \in \mathcal{U},\|u\|<c, \nu>N$, and for given sufficiently small $a_{l}^{-}, a_{r}^{+} \in \mathbb{R}^{n}$ there are $b \in Y_{\gamma} \times Y_{\gamma}$ and $a_{l}^{+}, a_{r}^{-} \in \mathbb{R}^{n}$ such that

$\left(B_{l}^{-}\right)\left(i d-P_{l}^{-}\left(u_{l}, \lambda\right)\left(-\omega^{-}\right)\left(v_{l}^{-}\left(a_{l}, u_{l}, \lambda\right)\left(-\omega^{-}\right)-a_{l}^{-}\right)=0\right.$,

$\left(B_{r}^{+}\right)\left(i d-P_{r}^{+}\left(u_{r}, \lambda\right)\left(\omega^{+}\right)\left(v_{r}^{+}\left(a_{r}, u_{r}, \lambda\right)\left(\omega^{+}\right)-a_{r}^{+}\right)=0\right.$,

$(C) v_{l}^{+}\left(a_{l}, u_{l}, \lambda\right)\left(\Omega^{+}\right)=w^{+}\left(b, u_{l}, \lambda\right)(0), \quad v_{r}^{-}\left(a_{r}, u_{r}, \lambda\right)\left(-\Omega^{-}\right)=w^{-}\left(b, u_{r}, \lambda\right)(0)$.

Proof. We show that $\left(a_{l}^{+}, a_{r}^{-}, b^{+}, b^{-}\right)$are uniquely determined in

$\Delta_{u, \lambda}:=\operatorname{im}\left(\mathrm{id}-P_{l}^{+}\left(\Omega^{+}\right)\right) \times \operatorname{im}\left(\mathrm{id}-P_{r}^{-}\left(-\Omega^{-}\right)\right) \times \operatorname{im} Q^{+}(0) \times \operatorname{im} Q^{-}(0)$.

Note that all projections appearing in the definition of $\Delta_{u, \lambda}$ depend on $\left(u_{l}, \lambda\right)$ or $\left(u_{r}, \lambda\right)$, respectively. Throughout the proof we suppose that $\left(a_{l}^{+}, a_{r}^{-}, b^{+}, b^{-}\right)$belongs to $\Delta_{u, \lambda}$.

We construct $a_{l}^{+}, a_{r}^{-}$and $b$ by solving an appropriate fixed point equation.

As a consequence of hypothesis 3.4 we know that $v_{l}^{+}\left(a_{l}, u_{l}, \lambda\right)\left(\Omega^{+}\right) \in Y_{\gamma}$. In what follows we suppress the dependence on $a_{l}, u_{l}, \lambda$ and $b$ from our notation. Therefore, in accordance with lemma 3.8, we find:

$$
\begin{aligned}
v_{l}^{+}\left(\Omega^{+}\right) & =Q^{+}(0) v_{l}^{+}\left(\Omega^{+}\right)+\left(\mathrm{id}-Q^{+}(0)\right) v_{l}^{+}\left(\Omega^{+}\right) \\
& =\left(\left.P_{s, l}^{+}\left(\Omega^{+}\right)\right|_{\operatorname{im} Q^{+}(0)}\right)^{-1} P_{s, l}^{+}\left(\Omega^{+}\right) v_{l}^{+}\left(\Omega^{+}\right)+\left(\mathrm{id}-P_{l}^{+}\left(\Omega^{+}\right) v_{l}^{+}\left(\Omega^{+}\right)\right. \\
& =\left(\left.P_{s, l}^{+}\left(\Omega^{+}\right)\right|_{\operatorname{im} Q^{+}(0)}\right)^{-1} a_{\perp, l}^{+}+a_{l}^{+} .
\end{aligned}
$$

On the other hand, in accordance with lemma 3.5.

$$
w^{+}(0)=Q^{+}(0) w^{+}(0)+\left(\mathrm{id}-Q^{+}(0)\right) w^{+}(0)=b^{+}+b_{\perp}^{+} .
$$

Hence, $v_{l}^{+}\left(a_{l}, u_{l}, \lambda\right)\left(\Omega^{+}\right)=w^{+}\left(b, u_{l}, \lambda\right)(0)$ if and only if

$$
b^{+}=\left(\left.P_{s, l}^{+}\left(\Omega^{+}\right)\right|_{\operatorname{im} Q^{+}(0)}\right)^{-1} a_{\perp, l}^{+}=: \alpha_{\perp, l}^{+} \quad \text { and } \quad a_{l}^{+}=b_{\perp}^{+} .
$$

In a similar way we find that $v_{r}^{-}\left(a_{r}, u_{r}, \lambda\right)\left(-\Omega^{-}\right)=w^{-}\left(b, u_{r}, \lambda\right)(0)$ if and only if

$$
b^{-}=\left(\left.P_{u, r}^{-}\left(-\Omega^{-}\right)\right|_{\operatorname{im} Q^{-}(0)}\right)^{-1} a_{\perp, r}^{-}=: \alpha_{\perp, r}^{-} \quad \text { and } \quad a_{r}^{-}=b_{\perp}^{-} .
$$

Altogether, for fixed $u_{l}, u_{r}$ and $\lambda$ equations (51) and (52) are equivalent to the fixed point equation

$$
\begin{aligned}
\left(a_{l}^{+}, a_{r}^{-}, b^{+}, b^{-}\right) & =\left(b_{\perp}^{+}(b), b_{\perp}^{-}(b), \alpha_{\perp, l}^{+}\left(a_{l}\right), \alpha_{\perp, r}^{-}\left(a_{r}\right)\right) \\
& =: \mathcal{G}_{u, \lambda}\left(\left(a_{l}^{+}, a_{r}^{-}, b^{+}, b^{-}\right),\left(a_{l}^{-}, a_{r}^{+}\right)\right),
\end{aligned}
$$

where we consider $\mathcal{G}$ as a mapping

$$
\mathcal{G}_{u, \lambda}: \Delta_{u, \lambda} \times\left(\mathbb{R}^{n} \times \mathbb{R}^{n}\right) \rightarrow \Delta_{u, \lambda} .
$$


To solve the fixed point equation (53) we apply the Banach fixed point theorem. First we show that there is a $\mathcal{G}_{u, \lambda}\left(\cdot,\left(a_{l}^{-}, a_{r}^{+}\right)\right)$-invariant closed ball $B(0, \varepsilon) \subset \Delta_{u, \lambda}$. Then we prove that $\mathcal{G}_{u, \lambda}\left(\cdot,\left(a_{l}^{-}, a_{r}^{+}\right)\right)$is a contraction on $B(0, \varepsilon)$.

Let $\tilde{c}_{l}(K)$ and $\tilde{c}_{r}(K)$ be the constants according to (23). The subscripts $l$ and $r$ refer to $v_{l}$ and $v_{r}$, respectively. With the constant $\tilde{\mathfrak{c}}$ related to $w$, cf. lemma 3.5, we define

$$
\epsilon=\epsilon(K):=\min \left\{\tilde{c}_{l}(K), \tilde{c}_{r}(K), \tilde{\mathfrak{c}}\right\} .
$$

Now we fix some sufficiently large $K$. Then the estimates given in (24) and corollary 3.7 provide that $\Omega^{+}, \Omega^{-}$and $N$ can be chosen so large that for all $\nu>N$ and for all $\left|a_{l}^{-}\right|,\left|a_{r}^{+}\right|<\epsilon(K)$ the mapping $\mathcal{G}_{u, \lambda}\left(\cdot,\left(a_{l}^{-}, a_{r}^{+}\right)\right)$leaves the closed ball $B_{\Delta_{u, \lambda}}(0, \epsilon(K))$ invariant. This remains true also for all larger $\Omega^{+}, \Omega^{-}$and $N$.

Due to (25) and (41) the mapping $\mathcal{G}_{u, \lambda}\left(\cdot,\left(a_{l}^{-}, a_{r}^{+}\right)\right)$is also a contraction on $B_{\Delta_{u, \lambda}}(0, \epsilon(K))$ (with increased $\Omega^{ \pm}$and $N$, if necessary).

Corollary 3.10. Let the assumptions of lemma 3.9 hold. Then $\left(a_{l}^{+}, a_{r}^{-}, b^{+}, b^{-}\right)$depend smoothly on $\left(a_{l}^{-}, a_{r}^{+}, u, \lambda\right)$.

Proof. For fixed $(u, \lambda)$ the smooth dependence on $\left(a_{l}^{-}, a_{r}^{+}\right)$follows by applying the implicit function theorem at a solution of (53).

To prove the smooth dependence on $(u, \lambda)$ we redo the proof of lemma 3.9 to some extent. This time, however, we decompose $v_{l}^{+}\left(\Omega^{+}\right)$and $w^{+}(0)$ by means of $Q^{+}(0,0)(0)$ instead of $Q^{+}\left(u_{l}, \lambda\right)(0)$. Similarly, we decompose $v_{r}^{-}\left(-\Omega^{-}\right)$and $w^{-}(0)$ by means of $Q^{-}(0,0)(0)$. In this way we get a fixed point equation in

$$
\Delta:=\operatorname{im}\left(\mathrm{id}-Q^{+}(0)\right) \times \operatorname{im}\left(\mathrm{id}-Q^{-}(0)\right) \times \operatorname{im} Q^{+}(0) \times \operatorname{im} Q^{-}(0),
$$

where all projections are considered at $\left(u_{l}, \lambda\right)=(0,0)$ or $\left(u_{r}, \lambda\right)=(0,0)$, respectively. Note that $\Delta$ does not depend on $(u, \lambda)$, and there is a $(u, \lambda)$-dependent isomorphism acting between $\Delta_{u, \lambda}$ and $\Delta$. This leads to a fixed point equation, similar to (53), defined by a mapping

$$
\mathcal{G}: \Delta \times\left(\mathbb{R}^{n} \times \mathbb{R}^{n}\right) \times \mathcal{U} \times \mathbb{R}^{m} \rightarrow \Delta .
$$

Exploiting this fixed point equation yields the corollary.

Proof of corollary 3.2. The statement of corollary 3.2 follows immediately from lemma 3.9 with $a_{l}^{-}=a_{r}^{+}=0$, see also the proof of corollary 2.8 and remark 2.9,

\subsection{Jump estimates}

Let the conditions of theorem 3.1 hold, and let $\left(x_{l}, x_{m}, x_{r}\right)$ denote the long Lin orbit segment. According to (26) we define:

$$
\begin{aligned}
& \Xi_{l}\left(\nu, a_{l}^{-}, a_{r}^{+}, u, \lambda\right):=x_{l}\left(\nu, a_{l}^{-}, a_{r}^{+}, u, \lambda\right)(0)-x_{m}\left(\nu, a_{l}^{-}, a_{r}^{+}, u, \lambda\right)(0), \\
& \Xi_{r}\left(\nu, a_{l}^{-}, a_{r}^{+}, u, \lambda\right):=x_{m}\left(\nu, a_{l}^{-}, a_{r}^{+}, u, \lambda\right)(\tau)-x_{r}\left(\nu, a_{l}^{-}, a_{r}^{+}, u, \lambda\right)(0) .
\end{aligned}
$$


We now consider exemplarily the jump $\Xi_{l}$ within $\Sigma_{l}$ more closely. For that purpose we write $\Xi_{l}$ in the form, cf. (27) and (28),

$$
\Xi_{l}\left(\nu, a_{l}^{-}, a_{r}^{+}, u, \lambda\right)=\xi_{l}^{\infty}\left(u_{l}, \lambda\right)+\xi_{l}\left(\nu, a_{l}^{-}, a_{r}^{+}, u, \lambda\right),
$$

where

$$
\begin{array}{ll}
\xi_{l}^{\infty}\left(u_{l}, \lambda\right) & :=q_{l}^{-}\left(u_{l}, \lambda\right)(0)-q_{l}^{+}\left(u_{l}, \lambda\right)(0), \\
\xi_{l}\left(\nu, a_{l}^{-}, a_{r}^{+}, u, \lambda\right):=v_{l}^{-}\left(a_{l}, u, \lambda\right)(0)-v_{l}^{+}\left(a_{l}, u, \lambda\right)(0),
\end{array}
$$

with $a_{l}=\left(a_{l}^{-}, a_{l}^{+}\left(a_{l}^{-}, a_{r}^{+}, u, \lambda\right)\right)$; cf. lemma 3.9.

Recall that we denote the leading stable Floquet multiplier of $\gamma$ by $\mu^{s}$.

Lemma 3.11. Let the constants $a_{l}^{-}, a_{r}^{+}, u$ and $\lambda$ be in agreement with theorem 3.1, and let $\nu$ be sufficiently large. Further, we assume hypotheses 2.1, 2.2 and 2.3 hold for the short Lin orbit segment defined by $q_{l}^{-1+}$ and $v_{l}^{-/+}$, and we assume that the non-orbit-flip condition for $q_{l}^{+}$holds, meaning that $q_{l}^{+}$is not in the strong stable manifold of $\gamma$. Then

$$
\xi_{l}\left(\nu, a_{l}^{-}, a_{r}^{+}, u, \lambda\right)=\mathcal{O}\left(\left(\mu^{u}\right)^{-\nu}\right)+\mathcal{O}\left(\left|a_{l}^{-}\right|\right) .
$$

The $\mathcal{O}(\cdot)$-terms are valid for $\nu \rightarrow \infty$ or $\left|a_{l}^{-}\right| \rightarrow 0$, respectively.

Proof. Lemma 2.10 yields that $\xi_{l}\left(\nu, a_{l}^{-}, a_{r}^{+}, u, \lambda\right)=\mathcal{O}\left(\left|a_{l}^{-}\right|\right)+\mathcal{O}\left(\left|a_{l}^{+}\right|\right)$. The coupling condition $(C)$, see also (53), yields that $a_{l}^{+}=b_{\perp}^{+}$, and from lemma 3.6 we get $\left|b_{\perp}^{+}\right|=c^{u}(b, u, \lambda)\left(\mu^{u}\right)^{-\nu}+o\left(\left|\mu^{u}\right|^{-\nu}\right)$.

In what follows we assume that $x_{l}$ and $x_{r}$ approach $\gamma_{l}$ and $\gamma_{r}$, respectively. For the jumps of the heteroclinic Lin orbit $x_{l} \cup x_{m} \cup x_{r}$ connecting $\gamma_{l}$ and $\gamma_{r}$ (via $\gamma$ ) we get:

Corollary 3.12. Let the heteroclinic Lin orbit $x_{l} \cup x_{m} \cup x_{r}$ be in agreement with corollary 3.2, and let $\nu$ be sufficiently large. Further we assume hypotheses [2.1, 2.2 and 2.3 hold for the short Lin orbit segment defined by $q_{l}^{-1+}$ and $v_{l}^{-1+}$, and we assume that the non-orbit-flip condition for $q_{l}^{+}$holds, meaning that $q_{l}^{+}$is not in the strong stable manifold of $\gamma$. Then there is a smooth function $c_{l}^{u}: \mathcal{U} \times \mathbb{R}^{m} \rightarrow \mathbb{R}$ such that

$$
\left\langle z, \xi_{l}(\nu, u, \lambda)\right\rangle=c_{l}^{u}(u, \lambda)\left(\mu^{u}\right)^{-\nu}+o\left(\left|\mu^{u}\right|^{-\nu}\right),
$$

where the $o(\cdot)$-term is valid for $\nu \rightarrow \infty$.

Proof. In accordance with corollary 2.11 and (29) we have

$$
\left\langle z, \xi_{l}(\nu, u, \lambda)\right\rangle=-\left\langle\Phi_{l}^{+}\left(0, \omega^{+}\right)^{T}\left(\mathrm{id}-P_{l}^{+}(u, \lambda)(0)\right)^{T} z, a_{l}^{+}\right\rangle+o\left(\left|a_{l}^{+}\right|\right) .
$$

Using (53) and (40) (in this order) yields

$$
\begin{aligned}
\left\langle z, \xi_{l}(\nu, u, \lambda)\right\rangle= & -\left\langle\Phi_{l}^{+}\left(0, \omega^{+}\right)^{T}\left(\mathrm{id}-P_{l}^{+}(u, \lambda)(0)\right)^{T} z, b_{\perp}^{+}\right\rangle+o\left(\left|b_{\perp}^{+}\right|\right) \\
= & -c^{u}(b, u, \lambda)\left(\mu^{u}\right)^{-\nu}\left\langle\Phi_{l}^{+}\left(0, \omega^{+}\right)^{T}\left(\mathrm{id}-P_{l}^{+}(u, \lambda)(0)\right)^{T} z, \hat{b}_{\perp}^{+}\right\rangle \\
& +o\left(\left(\mu^{u}\right)^{-\nu}\right)
\end{aligned}
$$

The notations $c^{u}$ and $\hat{b}_{\perp}^{+}$are in accordance with the proof of lemma 3.6. Note that both $b$ and $\hat{b}_{\perp}^{+}$depend on $(u, \lambda)$. With that we finally get

$$
c_{l}^{u}(u, \lambda)=-c^{u}(b, u, \lambda)\left\langle\Phi_{l}^{+}\left(0, \omega^{+}\right)^{T}\left(\mathrm{id}-P_{l}^{+}(u, \lambda)(0)\right)^{T} z, \hat{b}_{\perp}^{+}\right\rangle .
$$


The smoothness of $c_{l}^{u}$ follows from the representation (55) - recall that all ingredients there depend smoothly on $(u, \lambda)$.

Corollary 3.13. Let the assumptions of corollary 3.19 hold. Additionally let $n=3$. Then the function $c_{l}^{u}$ from corollary 3.12 satisfies $c_{l}^{u}(0,0) \neq 0$.

Proof. Consider the explicit representation (55) of $c_{l}^{u}$.

First we make clear that $c^{u}(0,0,0) \neq 0$ : For that we recall the arguments justifying (48) and note that, in the present context $\left(n=3\right.$ and hence $\left.\operatorname{dim} Y_{\gamma}=2\right)$, hypotheses 3.2 and 3.3 are automatically fulfilled.

Next we consider the second term on the right-hand side of (55)). Due to (2) and (4) we have (id $\left.-P_{l}^{+}(0,0)(0)\right)^{T} z=z$. Again due to $n=3$, and further due to the definitions of $b_{\perp}^{+}$and $Q^{+}$, cf. (39), and (31) it is clear that $\left\langle\Phi_{l}^{+}\left(0, \omega^{+}\right)^{T} z, \hat{b}_{\perp}^{+}\right\rangle \neq 0$.

Remark 3.14. The jump $\Xi_{r}$ in $\Sigma_{r}$ can be treated in a similar way.

\section{Application to EtoP cycles}

In this section we apply the theory of the existence of long Lin orbits developed in the previous sections to EtoP cycles. We discuss bifurcations of 1-homoclinic orbits to the equilibrium in the neighborhood of the EtoP cycle, and we compare these results with the numerical results of a concrete vector field from [11, 12, which also serves as the main motivating example for our studies. Here we refer to homoclinic orbits to $E$ (near the cycle under consideration) making only one excursion to $P$ as 1-homoclinic orbits.

Let the EtoP cycle consist of a hyperbolic equilibrium $E$, a hyperbolic periodic orbit $P$ and heteroclinic orbits $q_{l}$, connecting $E$ to $P$, and $q_{r}$ connecting $P$ to $E$. Then, in the language of the previous sections, such a EtoP cycle can be considered as an orbit segment $E \cup q_{l} \cup P \cup q_{r} \cup E$. The 1-homoclinic orbits to $E$ then can be found among the homoclinic Lin orbits near this orbit segment. Therefore, the bifurcation equations for detecting 1-homoclinic orbits to $E$ are generated by making the jump functions $\Xi_{l}$ and $\Xi_{r}$ equal to zero, cf. (54):

$$
\Xi_{l}(\nu, u, \lambda)=0, \quad \Xi_{r}(\nu, u, \lambda)=0 .
$$

Note that in the present context $a_{l}^{-}$and $a_{r}^{+}$are zero, the corresponding $\xi_{l}$ and $\xi_{r}$ are given by corollary 3.12 ,

In our analysis we distinguish two types of EtoP cycles. First, we consider codimension-one cycles characterized by a robust heteroclinic connection $q_{l}$ and a connection $q_{r}$ that splits up with positive speed while moving the family parameter $\lambda$. We prove an accumulation of 1-homoclinic orbits to $E$ near the original EtoP cycle in the following sense: for each sufficiently large $\nu \in \mathbb{N}$ there is a $\lambda_{\nu}$ for which a 1homoclinic orbit exists. This homoclinic orbit performs $\nu$ rotations along $P$ before returning to $E$. The $\lambda_{\nu}$ accumulate at $\lambda=0$, the critical parameter value for which the cycle exits. 
Second, we study codimension-two cycles, where in comparison with the previous one, we 'merely modify' the behavior near $q_{l}$ : We demand that the unstable manifold of $E$ and the stable manifold of $P$ have a quadratic tangency along $q_{l}$. The parameters $\lambda_{l}$ and $\lambda_{r}$ unfold the orbits $q_{l}$ and $q_{r}$ independently. Generically, 1-homoclinic orbits are still codimension-one objects - hence they are expected to appear along curves in the parameter space. Indeed, for each $\nu$ we find those orbits on a curve $\kappa_{\nu}$ in $\left(\lambda_{l}, \lambda_{r}\right)$ space. Each curve has a turning point which tends to the critical parameter value $\left(\lambda_{l}, \lambda_{r}\right)=(0,0)$. Here again $\nu$ counts the rotations of the 1-homoclinic orbits near $P$.

Our analysis is local in nature. However, the local phenomena described above are part of a global scenario observed numerically in several examples. In parameter space 1-homoclinic orbits can be continued along a curve which snakes between two curves (which are related to a 'quadratic tangency at $q_{l}$ ') and accumulates on a curve segment for which the EtoP cycle exists, an example will be introduced in the next section.

Finally we mention that in the recent paper [5] similar phenomena have been discussed.

\subsection{Unfolding of a saddle-node Hopf bifurcation with global reinjection mechanism}

In this section we consider a three-dimensional model vector field that was introduced in [11, the numerical results concerning the EtoP cycle presented here are from [12. The vector field describes the dynamics near a saddle-node Hopf bifurcation in the presence of a global reinjection mechanism. This type of dynamics with reinjection can be found, for example, in laser systems [13, 22, 23], in dynamo theory [1] and, more generally, near weak resonances [21, chapter 4.3.2]. The vector-field model can be written in the form

$$
\begin{aligned}
& \dot{x}=\Lambda_{1} x-\omega y-(\alpha x-\beta y) \sin \varphi-\left(x^{2}+y^{2}\right) x+d\left(2 \cos \varphi+\Lambda_{2}\right)^{2}, \\
& \dot{y}=\Lambda_{1} y+\omega x-(\alpha y+\beta x) \sin \varphi-\left(x^{2}+y^{2}\right) y+f\left(2 \cos \varphi+\Lambda_{2}\right)^{2}, \\
& \dot{\varphi}=\Lambda_{2}+s\left(x^{2}+y^{2}\right)+2 \cos \varphi+c\left(x^{2}+y^{2}\right)^{2},
\end{aligned}
$$

where $\Lambda_{1}$ and $\Lambda_{2}$ are the unfolding parameters of the saddle-node Hopf bifurcation. The parameters $\omega, \alpha, \beta, s, c, d$ and $f$ determine the type of unfolding and we keep them fixed throughout at

$$
\omega=1.0, \alpha=-1.0, \beta=0, s=-1.0, c=0, d=0.01, f=\pi d .
$$

This choice corresponds to the unfolding of type $A$ that was studied in [11, where more details can be found. The variable $\varphi$ is $2 \pi$-periodic and global reinjection is realized by trajectories that connect a neighborhood of a saddle-node Hopf point with one of its symmetric copies. Hence, a global reinjection corresponds to a large excursion near the circle $\mathbb{S}^{1}=\{x=y=0\}$. Note that this circle is not invariant because $d \neq 0$ and $f \neq 0$ (where rational ratios are avoided).

As already shown in [11], the system has a wide variety of homoclinic orbits of saddle-focus equilibria involving one or more global reinjections. The most interesting one in the present context is the homoclinic orbit $h_{1}$ to the saddle-focus equilibrium 
$E=\left(0,0, \arccos \left(\nu_{2} / 2\right)\right)$ with one global excursion, which accumulates on a curve segment in parameter space, while the orbit itself accumulates on an EtoP cycle connecting $E$ and a periodic orbit $P$. Figure 5 (a) shows the relevant part of the bifurcation diagram of (57) where the accumulation of $h_{1}$ takes place. Both curves $t_{0}$ are continuation curves of the codimension-one heteroclinic orbit $q_{l}$ connecting $E$ to a periodic orbit $P$. Here the codimension is characterized by a quadratic tangency of the unstable manifold of $E$ and the stable manifold of $P$. The curve $c_{1}$ is the continuation curve of the heteroclinic orbit $q_{r}$ connecting $P$ to $E$. The dimensions of the unstable manifold of $P$ and of the stable manifold of $E$ add up to the space dimension. Hence, $q_{r}$ is also a codimension-one heteroclinic orbit.

The complete heteroclinic EtoP cycle is given by $E \cup q_{l} \cup P \cup q_{r}$. Panels (b)-(e)
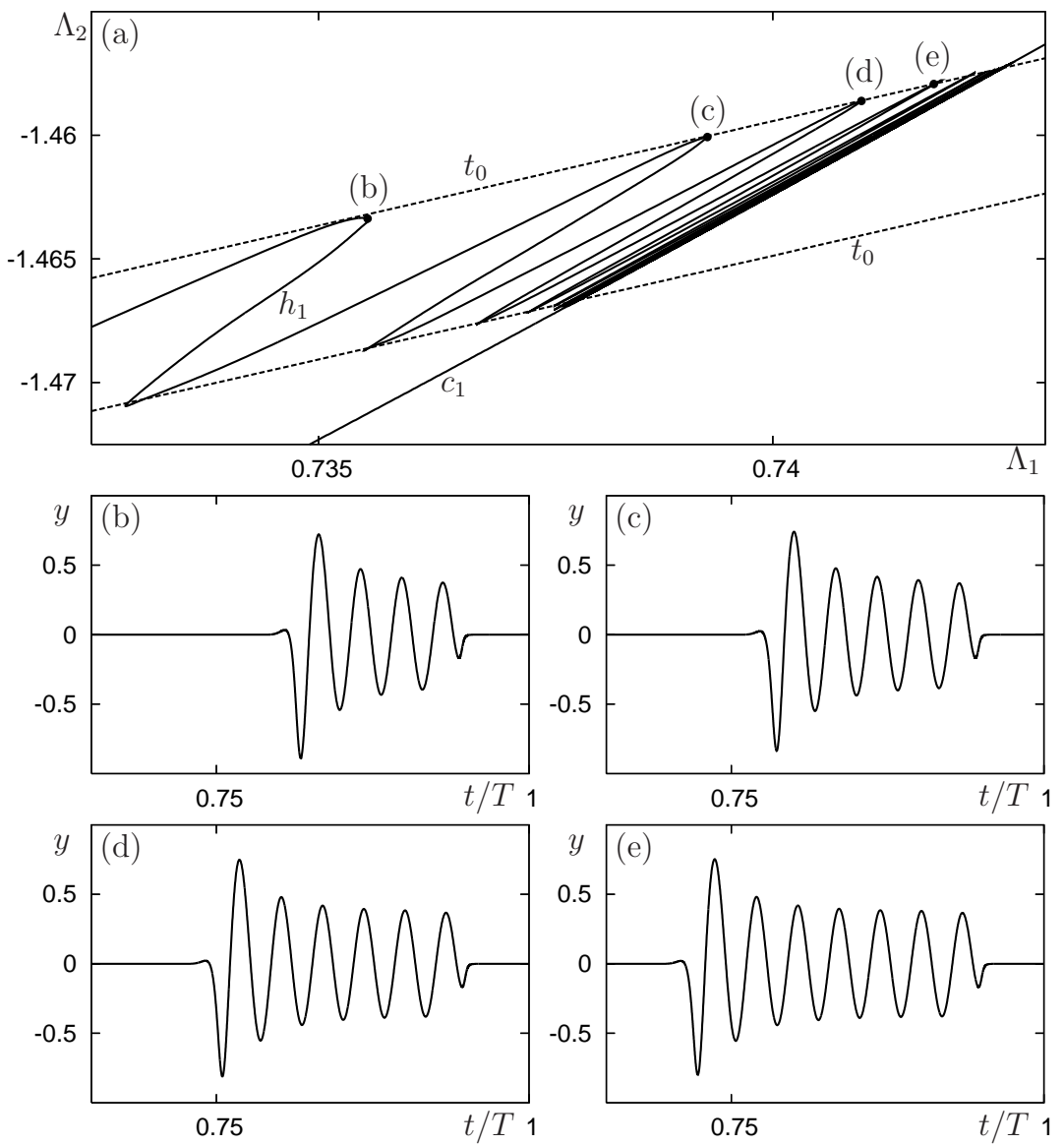

Figure 5. Panel (a) shows a detail of the bifurcation diagram of (57) in the $\left(\Lambda_{1}, \Lambda_{2}\right)$ plane. Shown are the curve $h_{1}$ of a codimension-one homoclinic orbit to $E$, the curve $c_{1}$ of a codimension-one EtoP connection from $E$ to $P$, and the curve $t_{0}$ of tangencies of a codimension-zero EtoP connection from $P$ back to $E$. Panels (b)-(e) show the relevant part of a time-versus- $y$ plot of selected homoclinic orbits on $h_{1}$ that illustrate how they take more rotations close to $P$ as they approach the complete EtoP cycle at the intersection of $c_{1}$ and $t_{0}$. Here, $T$ is the total integration time of the computed orbit segments. 
show time-versus- $y$ plots of selected homoclinic orbits along $h_{1}$ that illustrate how the homoclinic orbit accumulates on the EtoP cycle as the bifurcation curve $h_{1}$ accumulates on the segment of $c_{1}$ where the complete EtoP cycle exists.

Our goal here is to explain the accumulation process of $h_{1}$ analytically. More precisely, we are going to show two features of $h_{1}$. First, we consider a one-parameter family along a curve $\left(\Lambda_{1}(\lambda), \Lambda_{2}(\lambda)\right)$ somewhere in the middle between the two curves $t_{0}$, and show that the 1-homoclinic orbit $h_{1}$ to $E$ accumulates at discrete points on that parameter line. Second, using an unfolding of the tangencies $t_{0}$ at the intersection point of $t_{0}$ with $c_{1}$, we explain the shape of $h_{1}$ near the turning points (near the points labeled (b)-(e) in figure 5 (a)) during the snaking process.

\subsection{Accumulation of homoclinic orbits near EtoP cycles}

Consider a one-parameter family of ODE (1), that is $m=1$. We assume that for $\lambda=0$ there is a heteroclinic EtoP cycle consisting of a hyperbolic equilibrium $E$, a hyperbolic periodic orbit $P$ and heteroclinic orbits $q_{r}$ and $q_{l}$ connecting $P$ to $E$ and $E$ to $P$, respectively. In accordance with the notation in section section 3 , we have $E=\gamma_{l} \equiv \gamma_{r}$ and $P=\gamma$.

The aim of this section is to study homoclinic bifurcations from the given heteroclinic EtoP cycle under some additional genericity conditions.

Throughout we consider the system for $\lambda \in(-c, c), c$ sufficiently small. We assume:

(C1) $\operatorname{dim} W^{s}(E)=k$ and $\operatorname{dim} W^{u}(P)=n-k$;

(C2) $W^{s}(E)$ and $W^{u}(P)$ intersect in an isolated connecting orbit $q_{r}$;

(C3) The extended manifolds $\bigcup_{\lambda \in(-c, c)} W_{\lambda}^{u}(P) \times\{\lambda\}$ and $\bigcup_{\lambda \in(-c, c)} W_{\lambda}^{s}(E) \times\{\lambda\}$ intersect transversally in $\mathbb{R}^{n} \times \mathbb{R}$;

(C4) $W^{s}(P)$ and $W^{u}(E)$ intersect transversally along $q_{l}$;

(C5) The leading stable Floquet multiplier $\mu^{s}(\lambda)$ of $P$ is real and simple;

(C6) $q_{l}$ and $q_{r}$ approach $E$ and $P$ along the leading stable/unstable directions (nonorbit-flip condition).

Remark 4.1. The following one-parameter subfamily of (57) satisfies the conditions $(C 1)-(C 6)$ : Let $\kappa=\left(\Lambda_{1}(\lambda), \Lambda_{2}(\lambda)\right)$ and $\left(\Lambda_{1}(0), \Lambda_{2}(0)\right) \in \hat{c}_{1}$. Here $\hat{c}_{1}$ denotes the part of $c_{1}$ between the intersections of $c_{1}$ and the curves $t_{0}$. Further we assume that $\kappa$ and $c_{1}$ intersect transversally.

In order to apply the theory that we developed in the previous sections, we introduce cross-sections $\Sigma_{l / r}$ of $q_{l / r}$. Conditions $(\mathrm{C} 1)-(\mathrm{C} 4)$ ensure that $\operatorname{dim} U_{l}=\operatorname{dim} U_{r}=0$. Therefore, corollary 3.2 tells us that for each sufficiently small $\lambda$ there is a unique homoclinic Lin orbit $x=\left(x_{l}, x_{m}, x_{r}\right)$ connecting $E$ to itself. The actual 1-homoclinic orbits relate to solutions of the bifurcation equation (566). However, because of (C1)(C4) we have $\operatorname{dim} Z_{l}=0$ and $\operatorname{dim} Z_{r}=1$. This means that the (unique) homoclinic Lin orbit has exactly one discontinuity, and this discontinuity is located inside $\Sigma_{r}$. In other 
words, $\Xi_{l}$ is identically zero and the bifurcation equation for 1-homoclinic orbit reduces to (note that no $u$ is involved)

$$
\Xi_{r}(\nu, \lambda)=0 \text {. }
$$

The jump function $\Xi_{r}$ is defined by (26) and (27)

$$
\Xi_{r}(\nu, \lambda):=x_{m}(\nu, \lambda)(\tau)-x_{r}(\nu, \lambda)(0)=\xi_{r}^{\infty}(\lambda)+\xi_{r}(\nu, \lambda)
$$

Condition (C3) yields that the manifolds $W^{u}(P)$ and $W^{s}(E)$ split with non-vanishing velocity, in other words, $D \xi_{r}^{\infty}(0) \neq 0$. Hence there is a parameter transformation such that

$$
\xi_{r}^{\infty}(\lambda)=\lambda
$$

For the remaining term $\xi_{r}(\nu, \lambda)$ we may employ corollary 3.12 :

$$
\xi_{r}(\nu, \lambda)=c_{r}^{s}(\lambda)\left(\mu^{s}\right)^{\nu}+o\left(\left|\mu^{s}\right|^{\nu}\right)
$$

Combining these terms yields the following lemma.

Lemma 4.2. Assume (C1)-(C6) and (58). Then the jump function $\Xi_{r}(\nu, \lambda)$ can be written as

$$
\Xi_{r}(\nu, \lambda)=\lambda+c_{r}^{s}(\lambda)\left(\mu^{s}(\lambda)\right)^{\nu}+o\left(\left|\mu^{s}(\lambda)\right|^{\nu}\right),
$$

where $c_{r}^{s}(\lambda): \mathbb{R} \rightarrow \mathbb{R}$ is smooth, and the $o(\cdot)$-term is valid for $\nu \rightarrow \infty$.

A direct consequence of this lemma is the following corollary:

Corollary 4.3. Under the assumptions of lemma 4.2 there is a constant $N \in \mathbb{N}$ such that for all $\nu \in \mathbb{N}, \nu>N$, there is a $\lambda_{\nu}$ such that $\Xi_{r}\left(\nu, \lambda_{\nu}\right)=0$. Moreover, $\lambda_{\nu}$ tends to 0 as $\nu \rightarrow \infty$.

Note that the zeros of $\Xi_{r}$ correspond to 1-homoclinic orbits to $E$. Hence, corollary 4.3 says that for each sufficiently large natural $\nu$ there is a 1-homoclinic orbit with $\nu$ rotations near $P$. Further, we see that in parameter space these orbit accumulate at $\lambda=0$ - in state space they accumulate onto the original EtoP cycle.

For more precise assertions we need to know that $c_{r}^{s}(0) \neq 0$, which is true if $n=3$, see in the proof of corollary 3.13 , Therefore we get:

Corollary 4.4. Let $n=3$. Assume further (C1)-(C6) and (58).

(i) If $\mu^{s}>0$, then there is a monotonically increasing/decreasing (if $c_{r}^{s}(0)>0 / c_{r}^{s}(0)<$ 0) sequence $\left(\lambda_{\nu}\right), \lambda_{\nu} \rightarrow 0$, such that $\Xi_{r}\left(\nu, \lambda_{\nu}\right)=0$.

(ii) If $\mu^{s}<0$, then there is an alternating sequence $\left(\lambda_{\nu}\right), \lambda_{\nu} \rightarrow 0$, such that $\Xi_{r}\left(\nu, \lambda_{\nu}\right)=0$.

The order in which $\lambda_{\nu}$ approaches 0 for $\nu \rightarrow \infty$ is given by $\mu^{\nu}$.

A subfamily of (57) as described in remark 4.1 is related to (i) of corollary 4.4 . Altogether, in respect to (57) corollary 4.4 explains the accumulation process of $h_{1}$ along a line $\kappa$ according to remark 4.1. But it neither explains the global snaking behavior of $h_{1}$ nor the local behavior of $h_{1}$ near the turning points, cf. (b) - (e) in panel (a) of figure 5, which we consider in the following section. 


\subsection{Homoclinic orbits near degenerate EtoP cycles}

In this section we consider a codimension-two EtoP cycle. For that we modify in the formerly introduced EtoP cycle only the heteroclinic orbit $q_{l}$. Here we assume that along $q_{l}$ the unstable manifold of $E$ and the stable manifold of $P$ do no longer intersect transversally but have a quadratic tangency. We use parameters $\lambda_{l}$ and $\lambda_{r}$ to unfold the codimension-one heteroclinic orbits $q_{l}$ and $q_{r}$, respectively, and write $\lambda=\left(\lambda_{r}, \lambda_{l}\right) \in \mathbb{R}^{2}$. Here $\lambda_{r}$ plays the same role as $\lambda$ in the previous section, and $\lambda_{l}$ moves the manifolds $W^{u}(E)$ and $W^{s}(P)$ against each other in a direction which is orthogonal to the sum of the tanget spaces of these manifolds.

We consider system (1) for $|\lambda| \in(-c, c), c$ sufficiently small. In detail we assume the following: We adopt the assumptions (C1), (C2) and (C6) from the previous section as $\left(\mathrm{C}^{\prime}\right),\left(\mathrm{C} 2^{\prime}\right)$ and $\left(\mathrm{C}^{\prime}\right)$, respectively. In (C3) we only replace $\lambda$ by $\lambda_{r}$ :

(C3') the extended manifolds $\cup_{\lambda_{r} \in(-c, c)} W_{\left(\lambda_{r}, 0\right)}^{u}(P) \times\left\{\lambda_{r}\right\}$ and $\cup_{\lambda_{r} \in(-c, c)} W_{\left(\lambda_{r}, 0\right)}^{s}(E) \times\left\{\lambda_{r}\right\}$ intersect transversally in $\mathbb{R}^{n} \times \mathbb{R}$.

(C4') $W^{s}(P)$ and $W^{u}(E)$ have (along $q_{l}$ ) a quadratic tangency, and

the extended manifolds $\cup_{\lambda_{l} \in(-c, c)} W_{\left(0, \lambda_{l}\right)}^{u}(E) \times\left\{\lambda_{l}\right\}$ and $\cup_{\lambda_{l} \in(-c, c)} W_{\left(0, \lambda_{l}\right)}^{s}(P) \times\left\{\lambda_{l}\right\}$ intersect transversally in $\mathbb{R}^{n} \times \mathbb{R}$.

In contrast to (C5), here we also have an assumption on the leading unstable Floquet multiplier

(C5') the leading stable and unstable Floquet multipliers $\mu^{s / u}(\lambda)$ of $P$ are real and simple.

Remark 4.5. Consider 57). There is a parameter transformation $\left(\lambda_{r}, \lambda_{l}\right) \leftrightarrow\left(\Lambda_{1}, \Lambda_{2}\right)$, with $\left(\Lambda_{1}(0), \Lambda_{2}(0)\right) \in c_{1} \cap t_{0}$, such that in the new parameters (57) satisfies (C1') $\left(C 6^{\prime}\right)$.

The above assumptions imply the following dimensions of the involved linear subspaces: $\operatorname{dim} Z_{r}=1, \operatorname{dim} U_{r}=0, \operatorname{dim} Z_{l}=1, \operatorname{dim} U_{l}=1$. Hence, the variable $u=u_{l}$ appears in the jump function and in the bifurcation equation, respectively.

The jump function $\Xi$ now consists of two parts, each representing one jump:

$$
\Xi(\nu, u, \lambda)=\left(\begin{array}{c}
\Xi_{r}(\nu, u, \lambda) \\
\Xi_{l}(\nu, u, \lambda)
\end{array}\right),
$$

where $\Xi_{r / l}(\nu, u, \lambda)=\xi_{r / l}^{\infty}(u, \lambda)+\xi_{r / l}(\nu, u, \lambda)$. Indeed $\xi_{r}^{\infty}$ depends only on $\lambda_{r}$ - more precisely it has (after an appropriate parameter transformation) the form, see also (58),

$$
\xi_{r}^{\infty}\left(\lambda_{r}\right)=\lambda_{r}
$$

Further $\xi_{l}^{\infty}$ depends only on $\lambda_{l}$ and $u$, and the quadratic tangency within $\Sigma_{l}$ can be modeled by

$$
\xi_{l}^{\infty}\left(u, \lambda_{l}\right)=\lambda_{l}-u^{2}
$$

Altogether this yields 
Lemma 4.6. Assume $\left(C 1^{\prime}\right)-\left(C 6^{\prime}\right)$ and (60), (61). Then the bifurcation equation for 1-homoclinic orbits can be written as

$\Xi(\nu, u, \lambda)=\left(\begin{array}{c}\lambda_{r} \\ \lambda_{l}-u^{2}\end{array}\right)+\left(\begin{array}{c}c_{r}^{s}(u, \lambda)\left(\mu^{s}(\lambda)\right)^{\nu}+o\left(\left|\mu^{s}(\lambda)\right|^{\nu}\right) \\ c_{l}^{u}(u, \lambda)\left(\mu^{u}(\lambda)\right)^{-\nu}+o\left(\left|\mu^{u}(\lambda)\right|^{-\nu}\right)\end{array}\right)=0$,

where $c^{s / u}: \mathbb{R} \times \mathbb{R}^{2} \rightarrow \mathbb{R}$, are smooth and the o(.)-terms are valid for $\nu \rightarrow \infty$.
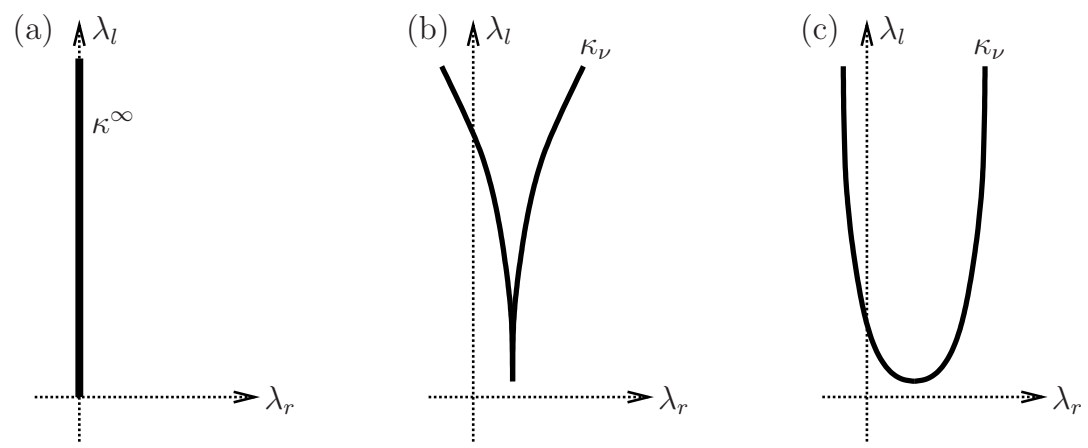

Figure 6. Solution curves of (63) and (62) in the $\left(\lambda_{r}, \lambda_{l}\right)$-plane for fixed $\nu$. Panel (a) shows the solution curve $\kappa^{\infty}$ of the unperturbed equation (63), which is a simple parabola in the $\left(\lambda_{l}, u\right)$-plane and a ray in the $\left(\lambda_{r}, \lambda_{l}\right)$-plane that is covered twice as $u$ is varied. Panels (b) and (c) show possible perturbations of $\kappa^{\infty}$.

Solutions of the 'unperturbed equation'

$$
\left(\begin{array}{c}
\lambda_{r} \\
\lambda_{l}-u^{2}
\end{array}\right)=0
$$

correspond to EtoP cycles near the original one. Solutions $\left(\lambda_{r}, \lambda_{l}\right)(u)$ of (63) are displayed in figure 6, panel (a). For each $\lambda_{l}>0$ there are two different heteroclinic orbits connecting $E$ to $P$. Therefore, for each nonzero $\lambda \in \kappa^{\infty}$ there are two different EtoP cycles satisfying $(\mathrm{C} 1)-(\mathrm{C} 6)$ (along a curve intersecting $\kappa^{\infty}$ transversally). So, according to corollary 4.3, we expect for fixed $\nu$ and $\lambda_{l}>0$ two different 1-homoclinic orbits $H_{1}\left(\nu, \lambda_{l}\right)$ and $H_{2}\left(\nu, \lambda_{l}\right)$ to $E$ with $\nu$ rotations near $P$. Both orbits can be continued in parameter space. Amazingly, they are located on the same continuation curve. Indeed, solutions of (62) for fixed $\nu$ are small perturbations of the solutions of (63). In parameter space we find those solutions on perturbations $\kappa_{\nu}$ of $\kappa^{\infty}$ as displayed in figure 6 (b) or (c). Both, $H_{1}\left(\nu, \lambda_{l}\right)$ and $H_{2}\left(\nu, \lambda_{l}\right)$ are on the same $\kappa_{\nu}$, but on different branches. For decreasing $\lambda_{l}$, the orbits $H_{1}\left(\nu, \lambda_{l}\right)$ and $H_{2}\left(\nu, \lambda_{l}\right)$ finally 'merge' in the vertex $\lambda_{\nu}$ of $\kappa_{\nu}$.

Generically one expects a perturbation of $\kappa^{\infty}$ as depicted in figure 6 (c). Below we show that in three-dimensional state space such a parabola like curve will indeed appear — as the numerical computations suggest.

Corollary 4.7. Let $n=3$. Assume further $\left(C 1^{\prime}\right)-\left(C 6^{\prime}\right)$ and (60), (61). For each (sufficiently large) $\nu \in \mathbb{N}$ there is a solution curve of $\kappa_{\nu}=\left(\lambda_{r}, \lambda_{l}\right)(u)$ of the bifurcation equation (62) for 1-homoclinic orbits. Further, there is a unique $u_{\nu}$ (for each $\nu$ ) such 
that $D \lambda_{l}\left(u_{\nu}\right)=0$ and $D \lambda_{r}\left(u_{\nu}\right) \neq 0$. The points $\lambda_{\nu}:=\left(\lambda_{r}, \lambda_{l}\right)\left(u_{\nu}\right)$ accumulate at $\lambda=0$, and the curvature of $\kappa_{\nu}$ in $\lambda_{\nu}$ tends to infinity as $\nu$ tends to infinity.

This corollary gives an explanation of the shape of $h_{1}$ (in figure 5 (a)) locally around the turning points. It also provides information about the exponential rates with which the turning points accumulate to $c_{1} \cap t_{0}$, namely they are given by the stable Floquet multiplier in the $\lambda_{r}$-direction, and by the unstable Floquet multiplier in the $\lambda_{l}$-direction. Similar numerical studies in [5] reveal that this mechanism also occurs in other systems.

A possible arrangement of curves $\kappa_{\nu}$ is displayed in figure 7. This picture verifies the shape of the curve $h_{1}$ in figure 5 near the points (b) - (e) analytically. The dashed line in figure 7 (the codimension-one line of the heteroclinic orbit $q_{l}$ ) corresponds to the upper curve $t_{0}$ in figure 5. Note that the vertices of the curves $\kappa_{\nu}$ are actually not located on this line. The fact that in figure 5 the points $(\mathrm{b})$ - (e) are seemingly on $t_{0}$ is due to the large absolute value of the unstable Floquet multiplier and the resulting quick convergence to $t_{0}$.

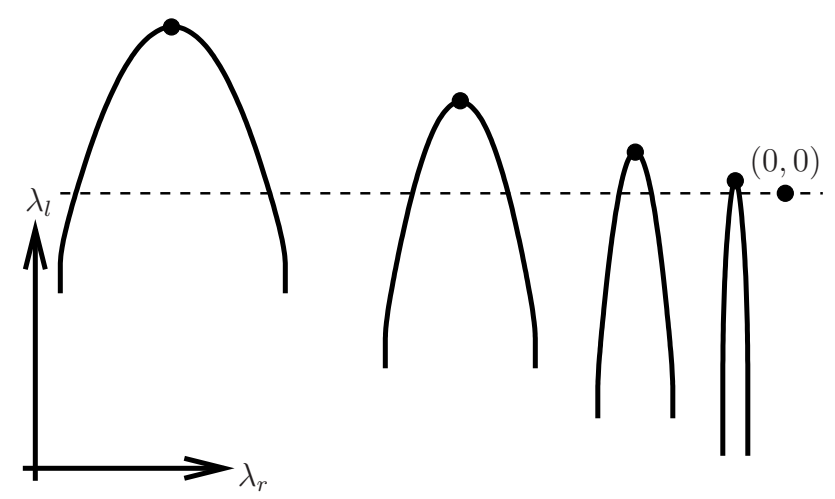

Figure 7. The parabolas $\Xi(\nu, u, \lambda)=0$ for increasing values of $\nu$ in the $\left(\lambda_{r}, \lambda_{l}\right)$-plane. The vertices of the parabolas approach $(0,0)$; the order of the displacement in the $\lambda_{r}$-direction is given by $\left(\mu^{s}\right)^{\nu}$ and the order of the displacement in the $\lambda_{l}$-direction is given by $\left(\mu^{u}\right)^{-\nu}$.

Proof. Consider the bifurcation equation (62). First we note that the derivatives of $\xi_{l / r}$ with respect to $u$ and $\lambda$ admit the same estimate as given in corollary 3.12 for $\xi_{l}$; we refer to [10] or [19] for similar assertions including proofs. So, using contraction arguments, we can solve $\Xi(\nu, u, \lambda)=0$ for $\kappa_{\nu}:=\lambda(u, \nu)$ for sufficiently large $\nu$. With

$$
D_{1} c_{r}^{s}(0,0) \neq 0
$$

follows the existence of vertices of $\kappa_{\nu}$. Again using (64) we can write $\kappa_{\nu}$ as $\lambda_{l}=\lambda_{l}\left(\lambda_{r}\right)$. From that representation one easily reads off the assertion concerning the curvature.

It remains to verify (64). Analogously to (55), we find

$$
c_{r}^{s}(u, \lambda)=c^{s}(b, u, \lambda)\left\langle\Phi_{r}^{-}\left(0,-\omega^{-}\right)^{T}\left(\mathrm{id}-P_{r}(\lambda)(0)\right)^{T} z, \hat{b}_{\perp}^{-}\right\rangle .
$$

Note that in the present context $(n=3)$ the scalar product on the right-hand side of (65) is different from zero, and the quantities within the scalar product do not depend 
on $u\left(\operatorname{dim} U_{r}=0\right)$. To verify that for $\hat{b}_{\perp}^{-}$recall the definition (39) of $b_{\perp}^{-}$. Note that $Q^{-}$ here does not depend on $u\left(\operatorname{dim} U_{r}=0\right)$ and $\operatorname{dimim}\left(\mathrm{id}-q^{-}(\lambda)\right)=1(n=3)$. Since $\hat{b}_{\perp}^{-}=b_{\perp}^{-} /\left|b_{\perp}^{-}\right|$we get that $\hat{b}_{\perp}^{-}$does depend neither on $u$ nor on $b$.

So $D_{1} c_{r}^{s}(0,0)$ is different from zero if and only if $D_{u} c^{s}(0,0,0) \neq 0$. Similarly to (48), we have $c^{s}(b, u, \lambda)=\left\langle\eta^{-}(b, u, \lambda), \eta^{s}(u, \lambda)\right\rangle$. Actually, here $\eta^{-}$does not depend on $(b, u)$. Further, note that $\eta^{-}$is related to the asymptotics of $\Psi^{-}\left(0,-\nu^{-}\right)\left(\mathrm{id}-Q^{-}(0)\right)^{T} \hat{b}_{\perp}^{-}$(see in the proof of lemma 3.6) and none of these terms depends on $u$. So $c^{s}=c^{s}(u, \lambda)$, and

$$
D_{1} c^{s}(0,0)=\left\langle\eta^{-}(0), D_{1} \eta^{s}(0,0)\right\rangle .
$$

Roughly speaking, $\eta^{s}(u, \lambda)$ is related to the asymptotics of $q_{d}^{+}(u, \lambda)(\cdot)$; see again the proof of lemma 3.6.

First we make clear that $D_{1} \eta^{s}(0,0)$ is different from zero. For this we assume that the traces of $W^{s}(P)$ in both $\Sigma_{l}$ and $\Sigma_{P}$ are flat (this can always be achieved by appropriate transformations). Then $W^{s}(P) \cap \Sigma_{l}$ coincides with $U$, and $q_{d}^{+}(u, 0)(0)=$ $\phi^{\Omega^{+}}(0)(u)$; compare hypothesis 3.4. Actually, this mapping can be considered as a mapping $\mathbb{R} \rightarrow \mathbb{R}$. Since $\phi^{\Omega^{+}}(0)(\cdot)$ is a diffeomorphism $\left(\mathbb{R}^{2} \rightarrow \mathbb{R}^{2}\right)$ also the above considered restriction to $U$ is a diffeomorphism, and

$$
D_{1} q_{d}^{+}(0,0)(0) \neq 0 .
$$

In the present context we have, see [10],

$$
\eta^{s}(u, \lambda)=\lim _{n \rightarrow \infty}\left(D_{1} \Pi(0, \lambda)\right)^{-n} q_{d}^{+}(u, \lambda)(n) .
$$

From that representation we conclude that with $D_{1} q_{d}^{+}(0,0)(0) \neq 0$ also $D_{1} \eta^{s}(0,0) \neq 0$.

Finally note that $\eta^{s}(u, 0) \in T_{\tilde{p}} W_{\Pi}^{s}(\tilde{p})=W^{s}(P) \cap \Sigma_{P}$ (see above, $\tilde{p}$ denotes the ח-equilibrium $\left.P \cap \Sigma_{P}\right)$, which is one-dimensional. Hence $D_{1} \eta^{s}(0,0)$ points in the same direction as $\eta^{s}(0,0)$ and, because of (66) ), we have $D_{1} c^{s}(0,0) \neq 0$ (see also the justification of (48)). Therefore (64) holds.

\section{Conclusions and outlook}

We adapted Lin's method to heteroclinic chains involving periodic orbits. The main emphasis was on the coupling of two short Lin orbits near a periodic orbit. In this way, we also achieved estimates of the jump functions (Lin gaps), which are essential for detecting actual orbits near the primary chain among the Lin orbits.

We employed our results to study 1-homoclinic orbits to the equilibrium near a given EtoP cycle. In particular we gave an analytical justification of some local phenomena in the course of the (global) snaking behavior of the continuation curve of 1-homoclinic orbits.

A complete analytical description of the snaking behavior is still an open problem; a global assumption on the behavior of the stable and unstable manifolds of $E$ and $P$, similar to that used in [2], is necessary for such an analysis. In [5] such an assumption 
has been used for a geometric explanation of the mentioned global snaking phenomenon. However, also these considerations are bound to $\mathbb{R}^{3}$.

Another interesting point is the more complete description of the dynamics near an EtoP cycle, such as existence of $N$-homoclinic (or $N$-heteroclinic) orbits to $E$ or $P$, periodic orbits, or shift dynamics.

\section{Acknowledgments}

The authors thank Bernd Krauskopf for helpful discussions and comments. TR acknowledges the financial support and hospitality of the Bristol Centre for Applied Nonlinear Mathematics at the Department of Engineering Mathematics, University of Bristol, during several long research visits and during a 6-month research position in 2008.

\section{References}

[1] Ashwin P, Rucklidge A M and Sturman R 2004 Two-state intermittency near a symmetric interaction of saddle-node and Hopf bifurcations: a case study from dynamo theory Physica D $19430-48$

[2] Beck M, Knobloch J, Lloyd D J B, Sandstede B and Wagenknecht T 2008 Snakes, ladders, and isolas of localised patterns. Accepted SIAM J. Math. Ana.

[3] Beyn W-J 1994 On well-posed problems for connecting orbits in dynamical systems Cont. Math. Chaotic Numerics $\mathbf{1 7 2} 131-68$

[4] Champneys A R, Kirk V, Knobloch E, Oldeman B E and Sneyd J 2007 When Shil'nikov Meets Hopf in Excitable Systems SIAM J. Appl. Dynam. Syst. 6 663-93

[5] Champneys A R, Kirk V, Knobloch E, Oldeman B E and Rademacher J D M 2008 Unfolding a tangent equilibrium-to-periodic heteroclinic cycle preprint

[6] Chow, S-N and Lin, X-B 1990 Bifurcation of a homoclinic orbit with a saddle-node equilibrium, J. Diff. and Int. Eq. 3(3) 435-466

[7] Coppel W A 1978 Dichotomies in stability theory Springer

[8] Deng B 1989 The Sil'nikov Problem, Exponential Expansion, Strong $\lambda$-Lemma, $C^{1}$-Linearization, and Homoclinic Bifurcation. J. Diff. Eq. 79 189-231

[9] Hale J and Lin X-B 1986 Heteroclinic Orbits for retarded Functional Differential Equations, J. Diff. Eq. 65 175-202

[10] Knobloch J 2004 Lin's Method for Discrete and Continuous Dynamical Systems and Applications Habilitationsschrift TU Ilmenau

[11] Krauskopf B and Oldeman B E 2006 Bifurcations of global reinjection orbits near a saddle-node Hopf bifurcation Nonlinearity 19 2149-67

[12] Krauskopf B and Rieß T 2008 A Lin's method approach to finding and continuing heteroclinic connections involving periodic orbits Nonlinearity 21 1655-90

[13] Krauskopf B, Tollenaar N and Lenstra D 1998 Tori and their bifurcations in an optically injected semiconductor laser Optics Communications 156 158-69

[14] Lin X-B 1990 Using Melnikov's method to solve Shilnikov's problems Proc. Roy. Soc. Edinburgh A $116295-325$

[15] Lin X-B 2008 Lin's method Scholarpedia 36972

[16] Rademacher J D 2005 Homoclinic orbits near heteroclinic cycles with one equilibrium and one periodic orbit J. Diff. Eq. 218 390-443 
[17] Rademacher J D 2008 Lyapunov-Schmidt reduction for unfolding heteroclinic networks of equilibria and periodic orbits with tangencies Preprint

[18] Rieß T 2008 A Lin's method approach to heteroclinic connections involving periodic orbits analysis and numerics Dissertationsschrift TU Ilmenau

[19] Sandstede B 1993 Verzweigungstheorie homokliner Verdopplungen Dissertationsschrift Universität Stuttgart

[20] Vanderbauwhede A and Fiedler B 1992 Homoclinic period blow-up in reversible and conservative systems ZAMP 43

[21] Vitolo R 2003 Bifurcations of attractors in 3D diffeomorphisms: a study in experimental mathematics PhD Thesis University of Groningen

[22] Wieczorek S M, Krauskopf B, Simpson T B and Lenstra D 2005 The dynamical complexity of optically injected semiconductor lasers Physics Reports 416 1-128

[23] Zimmermann M, Natiello M and Solari H 2001 Global bifurcations in a laser with injected signal: beyond Adler's approximation Chaos 11 500-13 